RICYDE. Revista Internacional de Ciencias del Deporte doi: $10.5232 /$ ricyde

Rev. int. cienc. deporte

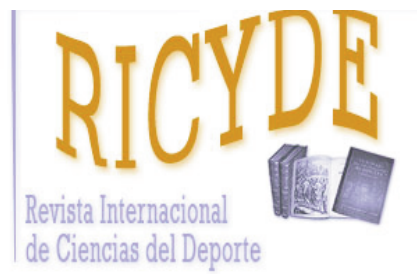

RICYDE. Revista Internacional de Ciencias del Deporte VOLUMEN XII - AÑO XII

Páginas:338-358 ISSN:1885-3137

Número 46 - Octubre - 2016

\title{
Altitud y deportes de equipo: métodos tradicionales desafiados por un entrenamiento innovador y específico en hipoxia \\ Altitude and team sports: traditional methods challenged by innovative sport-specific training in hypoxia
}

Franck Brocherie, Olivier Girard, Raphaël Faiss, Grégoire P. Millet

Institute of Sports Sciences, Faculty of Biology and Medicine, University of Lausanne, Switzerland

\section{Resumen}

Entre los diferentes métodos de entrenamiento en altitud, el "entrenamiento hipóxico intermitente" (IHT) método en el cual los atletas viven al nivel del mar pero se entrenan en condiciones de hipoxia - ha ganado una popularidad sin precedentes. Un análisis exhaustivo de los estudios que incluyen IHT muestra los beneficios sorprendentemente pobres para la mejora del rendimiento al nivel del mar, en comparación con el mismo entrenamiento realizado en normoxia. A pesar de las adaptaciones moleculares positivas observadas después de varias modalidades IHT, las características de estímulo del entrenamiento óptimo en hipoxia son todavía confusas y su traducción funcional en una mejora de rendimiento general es mínima. Para superar algunas limitaciones inherentes al IHT (carga de trabajo inferior debido a la hipoxia), estudios recientes han investigado un nuevo método, llamado RSH, de entrenamiento basado en la repetición de sprints con recuperaciones incompletas en hipoxia. Además, el creciente interés científico acerca de la aplicación práctica del entrenamiento hipóxico legitima el desarrollo de tecnologías innovadoras que sirven a los atletas de un deporte específico. Los objetivos de esta revisión son triples. Primero, analizar los resultados de los estudios que implican ejercicios de alta intensidad realizados en hipoxia para mejorar el rendimiento al nivel del mar diferenciando IHT y RSH. Segundo, discutir los posibles mecanismos que sustentan su eficacia y sus limitaciones inherentes. Tercero, presentar los beneficios potenciales del uso de innovaciones tecnológicas que indudablemente contribuirán a mejorar la comprensión de las adaptaciones fisiológicas inducidas por hipoxia mediante la realización de investigaciones pertinentes con un ajuste "ecológico" específico.

Palabras clave: entrenamiento en altitud; hipoxia; deportes intermitentes; sprints repetidos; ajuste ecológico.

\begin{abstract}
Among the different altitude training methods, intermittent hypoxic training (IHT); i.e., a method where athletes live at or near sea level but train under hypoxic conditions, has gained unprecedented popularity. A thorough analysis of studies including IHT, however, leads to strikingly poor benefits for sea-level performance improvement, compared to the same training method performed in normoxia. Despite positive molecular adaptations observed after various IHT modalities, the characteristics of optimal training stimulus in hypoxia are still unclear and their functional translation in term of whole-body performance enhancement is minimal. To overcome some of the inherent limitations of IHT (lower workload due to hypoxia), recent studies have successfully investigated a new training method based on the repetition of short sprints with incomplete recoveries in hypoxia; named RSH. Additionally, the growing scientific interest on the practical application of hypoxic training legitimizes the development of innovative technologies serving athletes in a sport-specific setting. The aims of the present review are therefore threefold. First, to critically analyze the results of the studies involving high-intensity exercises performed in hypoxia for sea-level performance enhancement by differentiating IHT and RSH. Second, to discuss the potential mechanisms underpinning their effectiveness and their inherent limitations. Third, to present the potentials benefits of using new technological innovation (i.e., the mobile inflatable simulated hypoxic system) which will undoubtedly contribute to the understanding advancement of hypoxia-induced physiological adaptations by conducting relevant research in the most sport-specific ecological test setting.
\end{abstract}

Key words: altitude training; hypoxia; intermittent sports; repeated sprints; ecological setting.

Correspondencia/correspondence: Franck Brocherie

ISSUL, Building Geopolis, Campus Dorigny, University of Lausanne, CH-1015, Lausanne, Switzerland

E-mail: franck.brocherie@unil.ch 
Brocherie, F.; Girard, O.; Fais, R., y Millet, G. P. (2016). Altitud y deportes de equipo: métodos tradicionales desafiados por un entrenamiento innovador y específico en hipoxia. RICYDE. Revista internacional de ciencias del deporte. 46(12), 338-358. http://dx.doi.org/10.5232/ricyde2016.04601

\section{Introducción}

L as estancias prolongadas en altitud que usan modelos "vivir arriba-entrenar arriba" (LHTH) o "vivir arriba-entrenar abajo" (LHTL) (Millet, Roels, Schmitt, Woorons y Richalet, 2010, Wilber, 2007) fueron populares entre los atletas de deportes de resistencia de larga duración y más recientemente entre los de deportes intermitentes (p.ej. deporte de equipo y de raqueta) con objetivo de generar una ventaja en competición (Billaut, Gore y Aughey, 2012, Millet, Faiss, Brocherie y Girard, 2013, Millet, y col., 2010). Sin embargo, debido a la interrupción de su entrenamiento y de su estilo de vida habitual, estas estrategias de estancias prolongadas en altitud geográfica natural (o hipobáricas) no son viables para todos los atletas de élite. Para simular los efectos físiológicos de la altitud, se han desarrollado últimamente nuevos dispositivos de hipoxia que funcionan con la disminución de la presión del aire inspirado (cámara hipobárica) o la reducción de la concentración de oxígeno $\left(\mathrm{O}_{2}\right)$ en el aire inspirado (por dilución con nitrógeno o filtración del $\mathrm{O}_{2} ; \mathrm{p}$. ej., tiendas de altitud, máquinas de hipoxia normobárica). Estos dispositivos han estimulado el desarrollo de una serie de procedimientos de entrenamiento derivado del método "vivir abajo-entrenar arriba" (LLTH); p. ej., "estancia intermitente hipóxica" (IHE) o "entrenamiento hipóxico intermitente" (IHT), para mejorar el rendimiento físico (con evaluación de atletas de resistencia sobre cinta ergométrica o cicloergómetro).

Por lo tanto, LLTH y métodos ulteriores de hipoxia simulada presentan la ventaja de implicar viajes mínimos, ser relativamente baratos y limitar la interrupción del estilo de vida y del medioambiente habitual de entrenamiento de los atletas. Otra ventaja es evitar el efecto nocivo (disminución de la excitabilidad muscular) de una exposición prolongada en altitud (Aughey, Clark, Gore, Townsend, Hahn, Kinsman, Goodman, Chow, Martin, Hawley y McKenna, 2006). Añadiendo el estrés hipóxico durante el entrenamiento de intervalo "aeróbico" o "anaerobia", se postula que el método reciente IHT mejora el rendimiento en comparación con un entrenamiento similar al nivel del mar. Durante mucho tiempo, la eritrocitosis era el factor principal que se beneficiaba de la mejora del rendimiento al nivel del mar después de un estímulo hipóxico suficiente (varias semanas) (Levine y Stray-Gundersen, 2006, Wilber, 2001, Wilber, Stray-Gundersen y Levine, 2007). Sin embargo, IHT ha abierto una nueva perspectiva evidente que muestra que ejerciendo en hipoxia, aunque sea con menor carga de trabajo, afecta a un gran número de genes mediados por factores inducibles por hipoxia (HIFs, "hypoxia-inducible factors") (Semenza, Shimoda y Prabhakar, 2006) y al rendimiento del ejercicio con adaptaciones musculares (y no necesariamente una mejora de la capacidad a transportar $\mathrm{O}_{2}$ ) (Hoppeler y Vogt, 2001, Lundby, Calbet y Robach, 2009, Vogt, Puntschart, Geiser, Zuleger, Billeter y Hoppeler, 2001, Zoll, Ponsot, Dufour, Doutreleau, Ventura-Clapier, Vogt, Hoppeler, Richard y Fluck, 2006). No obstante, en otros estudios sobre IHT, cualquier efecto potenciador de la hipoxia en el entrenamiento resultaba ambiguo (Bartsch, Dvorak y Saltin, 2009, Billaut, y col., 2012, Gatterer, Faulhaber y Netzer, 2009, Millet y Faiss, 2012, Morton y Cable, 2005). Aunque una mejora del rendimiento anaeróbico post-IHT ha sido mencionada en cuatro estudios (Daniels y Oldridge, 1970, Friedmann, Frese, Menold, Kauper, Jost y Bartsch, 2005, Gore, Hahn, Rice, Bourdon, Lawrence, Walsh, Stanef, Barnes, Parisotto, Martin y Pyne, 1998, Mizuno, Juel, Bro-Rasmussen, Mygind, Schibye, Rasmussen y Saltin, 1990), cabe destacar que estos estudios eran "incontrolados" y por lo tanto los efectos del entrenamiento no se pueden distinguir de los de la hipoxia (Bartsch, y col., 2009). Por tanto, parece que después de décadas de investigación "el IHT no aumenta el rendimiento físico al nivel del mar en atletas de resistencia más que el entrenamiento al nivel del mar" (Lundby, Millet, Calbet, Bartsch y Subudhi, 2012). 
Brocherie, F.; Girard, O.; Fais, R., y Millet, G. P. (2016). Altitud y deportes de equipo: métodos tradicionales desafiados por un entrenamiento innovador y específico en hipoxia. RICYDE. Revista internacional de ciencias del deporte. 46(12), 338-358. http://dx.doi.org/10.5232/ricyde2016.04601

Hasta el momento, existe escasa literatura que evalúe los beneficios potenciales del entrenamiento en altitud en deportes intermitentes (Brocherie, Girard, Faiss y Millet, 2015a, Galvin, Cooke, Sumners, Mileva y Bowtell, 2013, Gatterer, Philippe, Menz, Mosbach, Faulhaber y Burtscher, 2014, Hamlin, Marshall, Hellemans, Ainslie y Anglem, 2010a, Manimmanakorn, Hamlin, Ross, Taylor y Manimmanakorn, 2012, Manimmanakorn, Manimmanakorn, Taylor, Draper, Billaut, Shearman y Hamlin, 2013, Morton y Cable, 2005). A pesar de la creencia de que la estancia en altitud es una estrategia prometedora en deportes de equipo (Bartsch, Dvorak y Saltin, 2008, Bergeron, Bahr, Bartsch, Bourdon, Calbet, Carlsen, Castagna, Gonzalez-Alonso, Lundby, Maughan, Millet, Mountjoy, Racinais, Rasmussen, Singh, Subudhi, Young, Soligard y Engebretsen, 2012, Billaut, y col., 2012, Lundby, y col., 2012), los beneficios de la altitud sobre el rendimiento relacionado con partidos (capacidad de sprints repetidos, RSA) y los sistemas fisiológicos relevantes con atletas de deportes de equipo aún necesitan que se establezcan en protocolos de prueba más específicos de cada deporte (ajuste ecológico de prueba) (Brocherie, Millet, Hauser, Steiner, Rysman, Wehrlin y Girard, 2015c). Si bien los resultados de los protocolos bien diseñados en los límites de la "investigación aplicada" (p. ej., el resultado directamente aplicado en el ambiente deportivo) y a través de la "investigación fundamental" (p. ej., el enfoque mecánico) resultan útiles, la traducción del conocimiento científico a un entorno práctico es generalmente pobre (Crowley, 2003), especialmente debido a los problemas inherentes de la transferencia (p.ej., las actitudes conservadoras de entrenamiento, la formación técnica desactualizada) en aplicaciones deportivas profesionales. Protocolos bien diseñados utilizando condiciones específicas a los deportes de equipo (p.ej., para jugadores de fútbol, sobre césped natural con botas de fútbol) (Brocherie, Millet y Girard, 2015b, Girard, Racinais, Kelly, Millet y Brocherie, 2011) permiten una discusión mejor informada sobre cómo aplicar mejor las conclusiones de investigación en condiciones de campo y viceversa, sobre cómo los experimentos aplicados pueden ser utilizados para obtener una comprensión más profunda de los mecanismos inherentes. Para alcanzar estos objetivos, la innovación tecnológica se percibe generalmente como catalizadora para llevar a cabo investigaciones aplicadas relevantes en el ajuste ecológico más específico del deporte con el fin de cerrar la brecha entre ciencias, práctica y aplicación de programas de entrenamiento (Girard, Brocherie y Millet, 2013b).

Durante un partido, los jugadores de deportes de equipo (p.ej. fútbol) realizan un gran número de acciones de alta intensidad, incluyendo numerosos sprints con recuperaciones incompletas. Como consecuencia, el desarrollo de la capacidad a repetir ejercicios intensos durante períodos prolongados es importante para acciones cruciales en un partido (Iaia, Rampinini y Bangsbo, 2009). Por ejemplo, el hecho de recuperarse después de una secuencia de acciones intensas puede dejar al equipo más vulnerable en la defensa disminuyendo las posibilidades para alcanzar pases o aumentando el tiempo para tomar una posición defensiva ("tackles"). Junto con la innovación tecnológica (Girard, y col., 2013b), los métodos de entrenamiento específicos para deportes de equipo utilizando el estrés de hipoxia como fuerte estímulo adicional según modelos de LLTH/IHT expresamente diseñados son prometedores. Por ejemplo, el "entrenamiento de sprints repetidos en hipoxia" (RSH) definido como la repetición de varios períodos cortos $(\leq 30 \mathrm{~s})$ de ejercicio intenso ("all-out") en hipoxia intercalados con recuperaciones incompletas (proporción ejercicio-descanso de 1:4) podría considerarse como una estrategia del entrenamiento específico. 
Brocherie, F.; Girard, O.; Fais, R., y Millet, G. P. (2016). Altitud y deportes de equipo: métodos tradicionales desafiados por un entrenamiento innovador y específico en hipoxia. RICYDE. Revista internacional de ciencias del deporte. 46(12), 338-358. http://dx.doi.org/10.5232/ricyde2016.04601

Los objetivos de esta revisión son triples. Primero, analizar de manera critica los resultados de los estudios que implican ejercicios de alta intensidad realizados en hipoxia para mejorar el rendimiento al nivel del mar diferenciando IHT y RSH. Segundo, discutir los posibles mecanismos que sustentan la eficacia de esos métodos, sus limitaciones inherentes, junto con nuevas vías de investigación que rodean este tema. Tercero, presentar los beneficios potenciales del uso de esta innovación tecnológica (p. ej., el sistema de hipoxia simulada inflable móvil) que indudablemente contribuirá a mejorar la comprensión de las adaptaciones fisiológicas inducidas por hipoxia mediante la realización de investigaciones pertinentes en el mejor ajuste ecológico de prueba posible.

Recientemente, grupos de consenso internacionales (p. ej., IOC (Bergeron, y col., 2012), FIFA (Bartsch, Saltin y Dvorak, 2008) y paneles de expertos sobre entrenamiento en altitud para deportes de equipo (Girard, Amann, Aughey, Billaut, Bishop, Bourdon, Buchheit, Chapman, D'Hooghe, Garvican-Lewis, Gore, Millet, Roach, Sargent, Saunders, Schmidt y Schumacher, 2013a)) han subrayado la necesidad adicional "de estudiar los efectos del entrenamiento en hipoxia y modalidades LHTL sobre el rendimiento al nivel del mar, en altitudes baja y moderada con un diseño controlado por placebo con doble ciego". Siendo conscientes de las dificultades metodológicas [p. ej., la importancia de excluir efectos placebo (Bonetti y Hopkins, 2009, Lundby, y col., 2012)] - perteneciente a la conclusión de algunos estudios de entrenamiento en altitud - esta revisión se limita a los estudios que incluyen un grupo de control en su diseño experimental, permitiendo que los efectos del entrenamiento y del estímulo hipóxico se diferencien claramente.

\section{Tendencias actuales: ¿es el momento de avanzar más allá de IHT?}

\section{Mejora del rendimiento con IHT}

A día de hoy, hay 27 estudios controlados (20 IHT y sólo $7 \mathrm{RSH}$ ), incluyendo protocolos de entrenamiento realizados en hipoxia contra normoxia. Curiosamente, un beneficio adicional en el rendimiento del IHT comparado con el mismo entrenamiento realizado en normoxia está presente en sólo cuatro de esos estudios. Primero, Martino y col. (Martino, Myers y Bishop, 1995) reportaron tiempo más rápido en el 100-m de natación y una mayor mejora de la potencia máxima durante una prueba "Wingate" con brazos después de 21 días de entrenamiento que incluía sprints de natación a una altitud de $2800 \mathrm{~m}$, comparado con el nivel del mar. Ya que no existe una descripción detallada de las sesiones de entrenamiento, los mecanismos que inducen beneficios relacionados con la hipoxia adicional no pueden ser averiguados. Segundo, Hendriksen y Meeuwsen (2003) destacaron un aumento del 5\% de la potencia máxima durante una prueba de ciclismo "Wingate" después de 10 días de entrenamiento aeróbico en hipoxia hipobárica, mientras que el rendimiento en el grupo normóxico no cambió. Tercero, Dufour y col. (2006) relataron una capacidad de rendimiento a la resistencia mejorada en corredores de distancia después de 6 semanas de entrenamiento aeróbico de alta intensidad a $3000 \mathrm{~m} \mathrm{(5 \%} \mathrm{de} \mathrm{aumento} \mathrm{de} \mathrm{la} \mathrm{VO}_{2 \max }$ y $35 \%$ más tiempo hasta el agotamiento corriendo a una velocidad asociada a $\mathrm{VO}_{2 \max }$ ), pero no cambió el rendimiento para el grupo que se entrenaba en normoxia. Finalmente, Manimmanakorn y col. $(2012,2013)$ informaron en dos de los escasos estudios realizados con atletas de deportes de equipo que una extensión/flexión IHT de rodilla realizada durante un período de 5 semanas proporcionó un beneficio adicional para mejorar la contracción máxima voluntaria durante extensiones de pierna prolongadas. Una observación notable entre los estudios mencionados anteriormente consiste en que los beneficios adicionales de IHT parecen en parte relacionados con una regulación del potencial glucolítico y un aumento de la capacidad anaerobia (p. ej., mayor aumento de rendimiento al test "Wingate"). Estas adaptaciones podrían ayudar a atletas de deportes intermitentes para mejorar su rendimiento durante partidos. 
Brocherie, F.; Girard, O.; Fais, R., y Millet, G. P. (2016). Altitud y deportes de equipo: métodos tradicionales desafiados por un entrenamiento innovador y específico en hipoxia. RICYDE. Revista internacional de ciencias del deporte. 46(12), 338-358. http://dx.doi.org/10.5232/ricyde2016.04601

Además, en otro estudio conducido con atletas de deportes de equipo, se observaron mejoras similares en las dos potencias aeróbica y anaeróbica cuando el entrenamiento fue realizado en hipoxia y en normoxia (Morton y Cable, 2005). Otros estudios controlados y bien diseñados destacaron beneficios de IHT sobre el rendimiento aeróbico, pero fallaron en demostrar una ventaja adicional al hacer un entrenamiento en un entorno hipóxico (Katayama, Sato, Morotome, Shima, Ishida, Mori y Miyamura, 1999, Roels, Bentley, Coste, Mercier y Millet, 2007, Roels, Millet, Marcoux, Coste, Bentley y Candau, 2005, Roskamm, Landry, Samek, Schlager, Weidermann y Reindell, 1969, Truijens, Toussaint, Dow y Levine, 2003). Con diferentes estrategias y métodos de entrenamiento, la posibilidad de que el IHT pueda "mejorar el rendimiento de resistencia cuando el ejercicio ulterior es realizado en hipoxia" en jugadores de fútbol, como declarado en una reciente y amplia revisión (Billaut, y col., 2012), fue cuestionada por nuestro equipo (Millet y Faiss, 2012).

\section{Mecanismos fisiológicos y limitaciones del IHT}

El IHT probablemente tiene un efecto mínimo en la eritropoyesis ya que se requiere una gran "dosis hipóxica" para "estimular de manera significativa la vía eritropoyética hasta el punto en que mejora el rendimiento de resistencia al nivel del mar después de una exposición en altitud" (Levine y Stray-Gundersen, 2006, Wilber, y col., 2007). En apoyo de esta hipótesis, los anteriores estudios sobre IHT no lograron observar ningún cambio significativo en la masa de hemoglobina, en el volumen de glóbulos rojos o cualquier otro índice hematológico comparado con un grupo de control (Gore, Rodriguez, Truijens, Townsend, Stray-Gundersen y Levine, 2006, Holliss, Fulford, Vanhatalo, Pedlar y Jones, 2013) (ver (Millet, y col., 2010) para discusiones adicionales).

En comparación con el entrenamiento al nivel del mar, el IHT tiene el potencial de inducir un esfuerzo fisiológico adicional (Buchheit, Kuitunen, Voss, Williams, Mendez-Villanueva y Bourdon, 2012) y adaptaciones específicas moleculares (Kime, Karlsen, Nioka, Lech, Madsen, Saeterdal, Im, Chance y Stray-Gundersen, 2003, Vogt, y col., 2001, Zoll, y col., 2006), aunque no vayan necesariamente asociados con una mejora en la capacidad de ejercicio. El argumento para usar IHT se basa en la hipótesis que estas adaptaciones musculares sobrepasan los efectos provocados por el ejercicio normóxico. En particular, la presión parcial de $\mathrm{O}_{2}$ inferior en el tejido muscular durante IHT en comparación con el entrenamiento por intervalo conllevaría una mayor regulación al alza de HIF-1 $\alpha$ (Holliss, y col., 2013, Vogt, y col., 2001, Zoll, y col., 2006). Sin embargo, como se ha declarado recientemente (Lundby, y col., 2009), uno puede cuestionar que estas adaptaciones fisiológicas sean funcionalmente significativas (p.ej. el mayor aumento en la actividad del citrato sintasa en IHT que en el entrenamiento por intervalo), ya que los efectos del IHT sobre el rendimiento de resistencia medidos en normoxia son "mínimos e inconcluyente en atletas entrenados" (Lundby, y col., 2012).

Varios autores han reportado otras adaptaciones potencialmente favorables a los ejercicios de alta intensidad. Éstas incluyen: la mejora de la homeostasis del $\mathrm{O}_{2}$ en el músculo y de la perfusión tisular inducida por la mejora de la eficiencia mitocondrial, el control de la respiración mitocondrial (Ponsot, Dufour, Zoll, Doutrelau, N'Guessan, Geny, Hoppeler, Lampert, Mettauer, Ventura-Clapier y Richard, 2006, Roels, Thomas, Bentley, Mercier, Hayot y Millet, 2007), la angiogénesis (Toffoli, Roegiers, Feron, Van Steenbrugge, Ninane, Raes y Michiels, 2009) y la capacidad amortiguadora del músculo (Gore, Hahn, Aughey, Martin, Ashenden, Clark, Garnham, Roberts, Slater y McKenna, 2001). Sin embargo, la traducción en un mejor rendimiento no se observa en todos los casos y cuando ocurre, puede ser irrelevante para deportes de equipo. 
Brocherie, F.; Girard, O.; Fais, R., y Millet, G. P. (2016). Altitud y deportes de equipo: métodos tradicionales desafiados por un entrenamiento innovador y específico en hipoxia. RICYDE. Revista internacional de ciencias del deporte. 46(12), 338-358. http://dx.doi.org/10.5232/ricyde2016.04601

\section{Mejora del rendimiento con el entrenamiento de sprints repetidos en hipoxia}

Algunas de las limitaciones metodológicas relacionadas con IHT han sido superadas en los estudios recientes que investigan la nueva estrategia de entrenamiento hipóxico llamada entrenamiento de sprints repetidos en hipoxia (RSH) (Brocherie, y col., 2015a, Faiss, Leger, Vesin, Fournier, Eggel, Deriaz y Millet, 2013a, Faiss, Willis, Born, Sperlich, Vesin, Holmberg y Millet, 2015, Gatterer, y col., 2014, Kasai, Mizuno, Ishimoto, Sakamoto, Maruta y Goto, 2015, Puype, Van Proeyen, Raymackers, Deldicque y Hespel, 2013) (Tabla 1). RSH se basa en la repetición de esfuerzos "all-out" de corta ( $\leq 30 \mathrm{~s})$ duración intercalados con recuperaciones cortas e incompletas. Este modelo se diferencia del IHT en que la intensidad del estímulo del entrenamiento es máxima y por lo tanto permite el mantenimiento de un alto reclutamiento de fibras de contracción rápida (FT, "Fast-Twitch") de manera que cabe esperar resultados positivos añadiendo la hipoxia al entrenamiento. $\mathrm{RSH}$ es particularmente interesante ya que, en condiciones de hipoxia $(<3800 \mathrm{~m})$, generalmente se conserva el rendimiento de sprint único de corta duración $(<10 \mathrm{~s})$, mientras que la resistencia al cansancio durante pruebas de RSA se reduce antes y en mayor grado en el trabajo mecánico (Balsom, Gaitanos, Ekblom y Sjodin, 1994, Smith y Billaut, 2010).

Muy recientemente, nuestro grupo de investigación (Faiss, y col., 2013a) mostró que RSH retrasa el cansancio durante una prueba de RSA hasta al agotamiento (40\% de aumento del número de sprints completado). Esto fue confirmado en un otro estudio complementario realizado con esquiadores nórdicos de competición que realizaron 58\% más de sprints después de un entrenamiento RSH (Faiss, y col., 2015). RSH ha demostrado ser tan eficaz como RSN en la mejora de la potencia en un sprint único (5-7\%) cuando se incluyen sprints de 10-s intercalados con recuperaciones de 20-s (Faiss, y col., 2013a, Faiss, y col., 2015) o sprints de 30-s con recuperaciones de 270-s (Puype, y col., 2013), pero el cansancio solamente se retrasa durante RSA después del RSH (Faiss, y col., 2013a). Cabe destacar que, a pesar de mayores mejoras en el umbral anaeróbico después del entrenamiento hipóxico, ni Faiss y col. (2013a), ni Puype y col. (2013) han observado un incremento de rendimiento en pruebas de 3 min contrarreloj "all-out" y 10 min contrarreloj de ciclismo, respectivamente.

Además, la prueba al test Yo-Yo de recuperación intermitente de nivel 1 fue mejorada en un $33 \%$ después de 4 semanas de entrenamiento RSH sobre cinta ergométrica en jugadores de rugbi bien entrenados (Galvin, y col., 2013). Esto fue acompañado por una mayor distancia RSA recorrida después de un entrenamiento hipóxico, mientras que el tiempo total RSA mejoró de manera similar en ambos grupos hipóxico y normóxico (Galvin, y col., 2013). Más recientemente, Gatterer y col. (2014) han mostrado que el entrenamiento de velocidad especifica al fútbol es factible en cámaras hipóxicas de tamaño reducido $(4.75 \times 2.25 \mathrm{~m})$ y se asocia con un mejor mantenimiento de la velocidad de carrera durante una prueba de RSA (menor cansancio) en comparación con el mismo entrenamiento en normoxia. Por último, en futbolistas jóvenes bien preparados, añadir 10 sesiones de entrenamiento $\mathrm{RSH}$ en lugar de RSN (normoxia) a su práctica regular de fútbol durante un período de 5 semanas en temporada fue más eficiente en la mejora de la capacidad de agilidad repetida (incluyendo cambios de dirección), mientras que no tuvo ningún efecto adicional sobre las mejoras de la fuerza explosiva de las extremidades inferiores, de la velocidad máxima de carrera, del rendimiento RSA ni de la velocidad aeróbica máxima (Brocherie, y col., 2015a). 


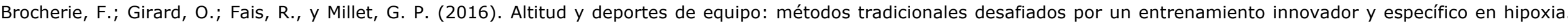
RICYDE. Revista internacional de ciencias del deporte. 46(12), 338-358. http://dx.doi.org/10.5232/ricyde2016.04601

Tabla 1: Resumen de los resultados de las actuales investigaciones en relación con el uso del entrenamiento de sprints repetidos en hipoxia

\begin{tabular}{|c|c|c|c|c|c|}
\hline $\begin{array}{l}\text { Autor } \\
\text { (año) }\end{array}$ & Participantes & $\begin{array}{l}\text { Diseño (número de sesiones de } \\
\text { entrenamiento; tipo; altitud; } \\
\text { contenido) }\end{array}$ & Pruebas de rendimiento & Grupos & Resultados principales \\
\hline \multirow{3}{*}{$\begin{array}{l}\text { Faiss y col. } \\
(2013 b)\end{array}$} & \multirow{3}{*}{$\begin{array}{l}\text { Ciclistas } \\
\text { moderadamente } \\
\text { entrenados }\end{array}$} & \multirow{3}{*}{$\begin{array}{l}8 \text { en } 4 \text { semanas; cicloergómetro; } \\
3000 \mathrm{~m}(\mathrm{NH}) ; 3 \times 510 \text {-s sprints } \\
\text { "all-out" intercalan con } 20 \text {-s de } \\
\text { recuperación a } 120 \mathrm{~W}\end{array}$} & \multirow{3}{*}{$\begin{array}{l}\text { RSA (10 s sprint, } 20 \mathrm{~s} \text { de } \\
\text { descanso hasta el agotamiento); } \\
\text { Sprint único de } 10-\mathrm{s} ; 3 \text { min "all- } \\
\text { out" contrarreloj; } 30-\mathrm{s} \\
\text { "Wingate" }\end{array}$} & $\mathrm{RSH}, \mathrm{N}=20$ & $\begin{array}{l}+\mathbf{3 8} \% \text { RSA sprints completos } \mathrm{y}+6 \% \\
\text { medio } \mathrm{PO} ;+10 \% \text { pico } \mathrm{PO} ; \mathrm{NS} \text { cambio } \\
\text { en medio } \mathrm{PO} ;+3 \% \text { medio } \mathrm{PO}\end{array}$ \\
\hline & & & & $\mathrm{RSN}, \mathrm{N}=20$ & $\begin{array}{l}\text { NS cambio en RSA sprints completos y } \\
+7 \% \text { medio PO }+6 \% \text { pico PO; NS } \\
\text { cambio en medio } \mathrm{PO} ;+5 \% \text { medio } \mathrm{PO}\end{array}$ \\
\hline & & & & $\mathrm{CON}, \mathrm{N}=10$ & NS cambios \\
\hline \multirow{3}{*}{$\begin{array}{l}\text { Puype y col. } \\
(2013)\end{array}$} & \multirow{3}{*}{$\begin{array}{l}\text { Ciclistas } \\
\text { moderadamente } \\
\text { entrenados }\end{array}$} & \multirow{3}{*}{$\begin{array}{l}18 \text { en } 6 \text { semanas; cicloergómetro; } \\
3000 \mathrm{~m}(\mathrm{NH}) ; 4-9 \times 30 \mathrm{~s} \text { sprints } \\
\text { intercalan con } 4.5 \mathrm{~min} \text { de } \\
\text { recuperación a } 50 \mathrm{~W}\end{array}$} & \multirow{3}{*}{$\begin{array}{l}\text { Medio PO de sprint único de } \\
\text { 30-s; } 10 \text {-min contrarreloj } \\
\text { simulado; test incremental }\end{array}$} & $\mathrm{RSH}, \mathrm{N}=10$ & $\begin{array}{l}+6 \% \text { sprint } \mathrm{PO} ;+6 \% 10 \text {-min } \mathrm{PO} ;+6 \% \\
\mathrm{VO}_{2 \max } \mathrm{y}+7 \% \text { LT4 }\end{array}$ \\
\hline & & & & $\mathrm{RSN}, \mathrm{N}=10$ & $\begin{array}{l}+5 \% \text { sprint } \mathrm{PO} ;+6 \% 10 \text {-min } \mathrm{PO} ;+6 \% \\
\mathrm{VO}_{2 \max } \text { y NS. }\end{array}$ \\
\hline & & & & $\mathrm{CON}, \mathrm{N}=10$ & NS cambios \\
\hline \multirow{2}{*}{$\begin{array}{l}\text { Galvin y col. } \\
(2013)\end{array}$} & \multirow{2}{*}{$\begin{array}{l}\text { Jugadores de } \\
\text { rugbi (academia) }\end{array}$} & \multirow{2}{*}{$\begin{array}{l}12 \text { en } 4 \text { semanas; cinta } \\
\text { ergométrica de correr; } 3500 \mathrm{~m} \\
(\mathrm{NH}) ; 10 \times 6 \text {-s sprint intercalan } \\
\text { con } 30 \mathrm{~s} \text { de recuperación }\end{array}$} & \multirow{2}{*}{$\begin{array}{l}\text { Yo-Yo IR } 1 ; 5-\mathrm{m} \text { sprint; RSA } \\
(10 \times 6-\mathrm{s}, 30 \mathrm{~s} \text { de descanso })\end{array}$} & RSH, N=15 & $\begin{array}{l}+33 \% \text { Yo-Yo IR1 distancia recorrida; } \\
-2 \% \text { tiempo de sprint; }+3.5 \% \text { distancia } \\
\text { total recorrida y }+\mathbf{2 7} \% \text { de decremento } \\
\text { de sprint }\end{array}$ \\
\hline & & & & RSN, N=15 & $\begin{array}{l}+14 \% \text { Yo-Yo IR } 1 \text { distancia recorrida; }- \\
2 \% \text { tiempo de sprint; }+1.5 \% \text { distancia } \\
\text { total recorrida y NS cambio en } \\
\text { decremento de sprint }\end{array}$ \\
\hline \multirow{2}{*}{$\begin{array}{l}\text { Gatterer y col. } \\
(2014)\end{array}$} & \multirow{2}{*}{$\begin{array}{l}\text { Jugadores de } \\
\text { futbol } \\
\text { (adolescentes) }\end{array}$} & \multirow{2}{*}{$\begin{array}{l}7-8 \text { en } 5 \text { semanas; carreras de ida } \\
\text { y vuelta; } 3000 \mathrm{~m}(\mathrm{NH}) ; 3 \times 510-\mathrm{s} \\
\text { esfuerzos "all-out" }(4.5 \mathrm{~m} \\
\text { carreras en naveta; } 20 \mathrm{~s} \text { y } 5 \text { min } \\
\text { de recuperaciones entre sprints y } \\
\text { series) }\end{array}$} & \multirow[t]{2}{*}{$\begin{array}{l}\text { Yo-Yo IR2; 5-m sprint; RSA (6 } \\
\times 40 \mathrm{~m}, 20 \mathrm{~s} \text { de descanso) }\end{array}$} & $\mathrm{RSH}, \mathrm{N}=5$ & $\begin{array}{l}+20 \% \text { Yo-Yo IR2; NS más rápido } 40-\mathrm{m} \\
\text { sprint, }+\mathbf{3 8 \%} \% \text { menor cansancio y }+28 \% \\
\text { decremento de sprint }\end{array}$ \\
\hline & & & & $\mathrm{RSN}, \mathrm{N}=5$ & $\begin{array}{l}+21 \% \text { Yo-Yo IR2; NS más rápido } 40 \mathrm{~m} \\
\text { sprint, }+9 \% \text { menor cansancio y }+10 \% \\
\text { decremento de sprint }\end{array}$ \\
\hline
\end{tabular}




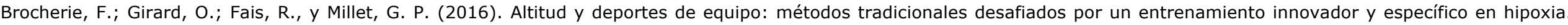
RICYDE. Revista internacional de ciencias del deporte. 46(12), 338-358. http://dx.doi.org/10.5232/ricyde2016.04601

Tabla 1: continuación.

\begin{tabular}{|c|c|c|c|c|c|}
\hline $\begin{array}{l}\text { Autor } \\
\text { (año) }\end{array}$ & Participantes & $\begin{array}{l}\text { Diseño (número de sesiones de } \\
\text { entrenamiento; tipo; altitud; } \\
\text { contenido) }\end{array}$ & Pruebas de rendimiento & Grupos & Resultados principales \\
\hline \multirow{2}{*}{$\begin{array}{l}\text { Faiss y col. } \\
(2015)\end{array}$} & \multirow{2}{*}{$\begin{array}{l}\text { Esquiadores } \\
\text { nórdicos Elite }\end{array}$} & \multirow{2}{*}{$\begin{array}{l}6 \text { en } 2 \text { semanas; "double-poling"; } \\
3000 \mathrm{~m}(\mathrm{NH}) ; 4 \times 510-\mathrm{s} \text { sprints "all- } \\
\text { out" intercalan con } 20-\mathrm{s} \text { de } \\
\text { recuperación a } 1 \mathrm{~W} \cdot \mathrm{kg}^{-1}\end{array}$} & \multirow{2}{*}{$\begin{array}{l}\text { RSA ( } 10 \text { s sprint, } 20 \mathrm{~s} \text { de } \\
\text { descanso hasta pico PO se redujo } \\
\text { en }>30 \% \text { ); Sprint único de } 10-\mathrm{s} \text {; } \\
\text { Sprints de equipo }(3 \times 3 \text { min "all- } \\
\text { out" con } 3 \text {-min de descanso) }\end{array}$} & $\mathrm{RSH}, \mathrm{N}=9$ & $\begin{array}{l}+\mathbf{5 7} \% \text { RSA sprints completos } \mathrm{y}+18 \% \\
\text { medio PO; }+22 \% \text { medio } \mathrm{PO} ;+11 \% \text { medio } \\
\text { PO }\end{array}$ \\
\hline & & & & $\mathrm{RSN}, \mathrm{N}=8$ & $\begin{array}{l}\text { NS cambios en RSA sprints completos y } \\
+22 \% \text { medio PO; }+27 \% \text { medio PO; }+15 \% \\
\text { medio PO }\end{array}$ \\
\hline \multirow{2}{*}{$\begin{array}{l}\text { Brocherie y } \\
\text { col. }(2015 \mathrm{a})\end{array}$} & \multirow{2}{*}{$\begin{array}{l}\text { Jugadores de } \\
\text { futbol } \\
\text { (adolescentes) }\end{array}$} & \multirow{2}{*}{$\begin{array}{l}10 \text { en } 5 \text { semanas; cinta ergométrica } \\
\text { de correr; } 2900 \mathrm{~m}(\mathrm{NH}) ; 5 \times 45-\mathrm{s} \\
\text { sprints "all-out" intercalan con } 45-\mathrm{s} \\
\text { de recuperación }\end{array}$} & \multirow{2}{*}{$\begin{array}{l}\text { RSA }(10 \times 30 \mathrm{~m}, 30 \mathrm{~s} \text { de } \\
\text { descanso); Agilitad repetido }(6 \times \\
20 \mathrm{~m}, 30 \mathrm{~s} \text { de descanso); tiempo } \\
\text { de sprint }(10-40 \mathrm{~m}) ; \mathrm{CMJ} \\
\text { VAMEVAL }\end{array}$} & $\mathrm{RSH}, \mathrm{N}=8$ & $\begin{array}{l}-3.0 \% \text { y }-3.2 \% \text { para los tiempos de sprint } \\
\text { inicial y medio; } \mathbf{- 4 . 4 \%} \text { y } \mathbf{- 4 . 3 \%} \text { parales } \\
\text { tiempos de sprint inicial y medio; }-1 \% \\
\text { tiempo de sprint; }+6.5 \% \text { PO; NS cambio en } \\
\text { la velocidad aeróbica máxima }\end{array}$ \\
\hline & & & & $\mathrm{RSN}, \mathrm{N}=8$ & $\begin{array}{l}-2.3 \% \text { y }-1.9 \% \text { para los tiempos inicial y } \\
\text { medio; }-2.0 \% \text { y }-2.4 \% \text { para los tiempos } \\
\text { inicial y medio; }-2 \% \text { tiempo de sprint; }+5.0 \% \\
\text { PO; NS cambio en la velocidad aeróbica } \\
\text { máxima }\end{array}$ \\
\hline \multirow[b]{2}{*}{$\begin{array}{l}\text { Kasai y col. } \\
(2015)\end{array}$} & \multirow[b]{2}{*}{$\begin{array}{l}\text { Jugadores de } \\
\text { Lacrosse } \\
\text { (femeninas) }\end{array}$} & \multirow[b]{2}{*}{$\begin{array}{l}8 \text { en } 4 \text { semanas; cicloergómetro; } 3000 \\
\mathrm{~m}(\mathrm{NH}) ; 2 \times 107 \text {-s sprints "all-out" } \\
\text { intercalan con } 30 \text {-s de recuperación }\end{array}$} & \multirow[b]{2}{*}{$\begin{array}{l}\text { RSA }(10 \times 7-\mathrm{s} \text { sprints, } 30-\mathrm{s}) \\
\mathrm{VO}_{2 \max }+\text { tiempo hasta el } \\
\text { agotamiento }(\mathrm{TTE})\end{array}$} & $\mathrm{RSH}, \mathrm{N}=16$ & $\begin{array}{l}+\mathbf{5 . 0} \% \text { pico PO; }+\mathbf{9 . 7 \%} \text { medio } \mathrm{PO} ;+3.0 \% \\
\mathrm{VO}_{2 \max } ;+\mathbf{4 . 4 \%} \text { TTE }\end{array}$ \\
\hline & & & & RSN, N=16 & $\begin{array}{l}+1.5 \% \text { pico } \mathrm{PO} ;+6.0 \text { medio } \mathrm{PO} ;+0.5 \% \\
\mathrm{VO}_{2 \max } ;+3.4 \% \text { TTE }\end{array}$ \\
\hline
\end{tabular}

Una diferencia significativa entre los grupos se muestra en negrita $(\mathrm{P}<0.05)$.

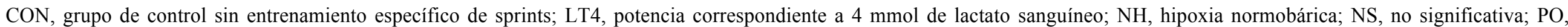

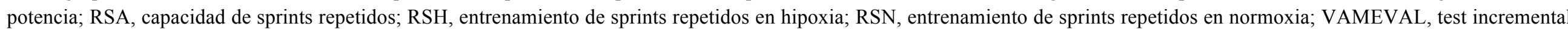

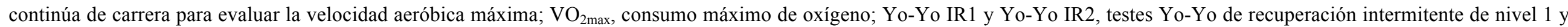
2 , respectivamente. 
Brocherie, F.; Girard, O.; Fais, R., y Millet, G. P. (2016). Altitud y deportes de equipo: métodos tradicionales desafiados por un entrenamiento innovador y específico en hipoxia. RICYDE. Revista internacional de ciencias del deporte. 46(12), 338-358. http://dx.doi.org/10.5232/ricyde2016.04601

En el estudio de Kasai y col. (2015), encontraron un efecto positivo del RSH respecto al RSN en mejorar la potencia máxima durante una prueba de RSA en atletas femeninos de lacrosse. Por consiguiente, como respaldan estos cuatro estudios sobre jugadores de deportes de equipo (Brocherie, y col., 2015a, Galvin, y col., 2013, Gatterer, y col., 2014, Kasai, y col., 2015), RSH puede producir supuestos beneficios para otros deportes de equipo y de raqueta en los que la capacidad de sprints repetidos durante todo un partido es esencial para el rendimiento general. Sin embargo, otros estudios en entornos ecológicamente validados están justificados para respaldar la eficacia del RSH en deportes de equipo y de raqueta.

\section{Mecanismos fisiológicos y perspectivas de RSH}

Planteamos la hipótesis de que RSH induciría adaptaciones beneficiosas principalmente por la mejora en el nivel de perfusión sanguínea que implica una mejor utilización del $\mathrm{O}_{2} \mathrm{y}$ un mejor comportamiento de las fibras FT. Con intensidades de esfuerzo máximas, las adaptaciones específicas del tejido muscular esquelético (al nivel molecular) pueden surgir a través de la vía de detección del $\mathrm{O}_{2}$ (p. ej., proporción capilar-a-fibra, área de la sección transversal de la fibra, el contenido de la mioglobina y la actividad enzimática oxidativa como el citrato sintasa) lo que no ocurre en condiciones de normoxia o, de ocurrir, es en un menor grado (Hoppeler y Vogt, 2001, Vogt, y col., 2001, Zoll, y col., 2006). Además, es conocido que hacer ejercicio en hipoxia provoca una vasodilatación compensatoria para responder a un aumento de la demanda de $\mathrm{O}_{2}$ a nivel muscular (Casey y Joyner, 2012).

Sprints repetidos causan grandes cambios en las concentraciones tanto de fosfocreatina ([PCr] $)$ como de los iones de hidrógeno $\left(\mathrm{H}^{+}\right)$. Sin embargo, la recuperación de la potencia durante sprints repetidos parece estar más influenciada por el suministro de energía muscular (p.ej. resíntesis de [PCr]) que por la recuperación de $\mathrm{pH}$ muscular (Mendez-Villanueva, Edge, Suriano, Hamer y Bishop, 2012). Sin embargo, el aumento de la capacidad de amortiguación o de regulación de los genes implicados en el control del $\mathrm{pH}$ también se han reportado después RSH (Faiss, y col., 2013a, Puype, y col., 2013).

Durante sprints en hipoxia, la vasodilatación compensatoria (con un aumento del flujo sanguíneo) que apunta al mantenimiento constante de la entrega total de $\mathrm{O}_{2}$ al músculo es probablemente máxima ya que la intensidad del ejercicio es esencial en la amplitud de este mecanismo compensatorio (Casey y Joyner, 2012). Las fibras FT son susceptibles de beneficiarse más que las fibras de contracción lenta (ST, "Slow-Twitch") por la alta perfusión sanguínea. A partir de estas conclusiones y debido a su mayor extracción de $\mathrm{O}_{2}$ fraccional (McDonough, Behnke, Padilla, Musch y Poole, 2005) la entrega de $\mathrm{O}_{2}$ microvascular mejorado para las fibras FT "haría que las fibras FT se comportarsen más como ST, que son más eficientes en la oxidación" (Cleland, Murias, Kowalchuk y Paterson, 2012). En este contexto, es interesante que los músculos de la parte superior del brazo contienen una alta proporción de FT (Klein, Marsh, Petrella y Rice, 2003). Por ejemplo, en jugadores de tenis profesional, $2 / 3$ de todas las fibras en el m. tríceps braquii son FT, mientras que el valor correspondiente para el m. vasto lateralis es sólo 1/3 (Sanchis-Moysi, Idoate, Olmedillas, Guadalupe-Grau, Alayon, Carreras, Dorado y Calbet, 2010). Muy recientemente, se recurrió a esquiadores nórdicos de élite (técnica "double-polling") para conocer la influencia del RSH en el rendimiento de los músculos superiores del cuerpo (Faiss, y col., 2015). La conclusión principal fue que RSH realizado en grupos musculares superiores indujo un mejor rendimiento del entrenamiento normóxico. Cabe destacar que la de/re-oxigenación del $\mathrm{m}$. tríceps braquii, que contiene una alta proporción de fibras FT, se midió para evaluar potenciales adaptaciones específicas del tipo de fibra provocadas por RSH. Los autores observaron una mayor amplitud de las variaciones en la perfusión sanguínea durante RSH en comparación con el mismo entrenamiento en normoxia (Faiss, y col., 2015). 
Brocherie, F.; Girard, O.; Fais, R., y Millet, G. P. (2016). Altitud y deportes de equipo: métodos tradicionales desafiados por un entrenamiento innovador y específico en hipoxia. RICYDE. Revista internacional de ciencias del deporte. 46(12), 338-358. http://dx.doi.org/10.5232/ricyde2016.04601

\section{Beneficios potenciales de la innovación tecnológica / futuras investigaciones}

En los últimos años, el interés creciente por la aplicación práctica del entrenamiento en altitud en deportes de equipo - principalmente en el fútbol - se debió a la necesidad de los equipos de lograr una aclimatación óptima para participar en la FIFA 2010 (Sudáfrica) y 2011 U-20 (Colombia) Copas Mundiales realizadas en altitud. A pesar de este aumento de interés por parte de las principales organizaciones deportivas, resulta llamativo que hasta hoy sólo haya diez estudios de entrenamiento en hipoxia (incluyendo los cuatros estudios RSH) realizados con jugadores de deportes de equipo (Brocherie, y col., 2015a, Brocherie, y col., 2015c, Galvin, y col., 2013, Gatterer, y col., 2014, Hamlin, Hinckson, Wood y Hopkins, 2008, Hamlin, Marshall, Hellemans, Ainslie y Anglem, 2010b, Kasai, y col., 2015, Manimmanakorn, y col., 2012, Manimmanakorn, y col., 2013, Morton y Cable, 2005). Por lo tanto, existe una necesidad urgente de estudios tanto mecánicos como aplicados que investiguen los cambios de rendimiento en deportes de equipo tras un entrenamiento hipóxico con poblaciones de deportes específicos antes de formular definitivamente recomendaciones basadas en evidencias robustas. Con el fin de cerrar la brecha entre el conocimiento científico y su aplicación práctica en un entorno ecológico específico del deporte de equipo, el desarrollo de nuevos dispositivos hipóxicos (p. ej., el sistema de hipoxia simulada inflable móvil) permite el desarrollo de protocolos aplicados bien diseñados. Tal material será útil para probar la eficacia de nuevos enfoques de entrenamiento en hipoxia.

\section{Innovación tecnológica: el sistema de hipoxia simulada inflable móvil}

Un nuevo equipo de hipoxia simulada inflable móvil (Altitude Training Systems - High Performance Unit, Australia) fue diseñado según las normas ISO (ISO 13485:2008) y fue mostrado por primera vez durante la conferencia "Altitude Training and Team Sports" (Marzo 2013, Doha, Qatar). El sistema tiene dos unidades hinchables - p. ej., un túnel y un diseño rectangular (Figura 1) - y un remolque hipóxico, que genera el aire hipóxico con fracción inspirada de $\mathrm{O}_{2}$ entre 0.21 y 0.10 (una altitud simulada hasta $5100 \mathrm{~m}$ ). Las especificaciones completas han sido descritas en otro documento (Girard, y col., 2013b). Este sistema hipóxico ofrece a atletas de deportes de equipo la capacidad de entrenarse en condiciones específicas de cada deporte con una variedad de actividades relacionadas con el rendimiento durante partidos y varias tareas (p.ej. sprints y simulaciones de carreras o pequeños juegos y entrenamiento de circuito, dependiendo de la unidad) (ver (Brocherie, y col., 2015c)). El uso de las instalaciones de hipoxia simulada con sistemas inflables móviles contribuirá indudablemente a promover la comprensión de las adaptaciones fisiológicas inducidas por hipoxia mediante la realización de investigaciones relevantes en entornos de prueba "ecológicos" lo más específicos de cada deporte. 
Brocherie, F.; Girard, O.; Fais, R., y Millet, G. P. (2016). Altitud y deportes de equipo: métodos tradicionales desafiados por un entrenamiento innovador y específico en hipoxia. RICYDE. Revista internacional de ciencias del deporte. 46(12), 338-358. http://dx.doi.org/10.5232/ricyde2016.04601

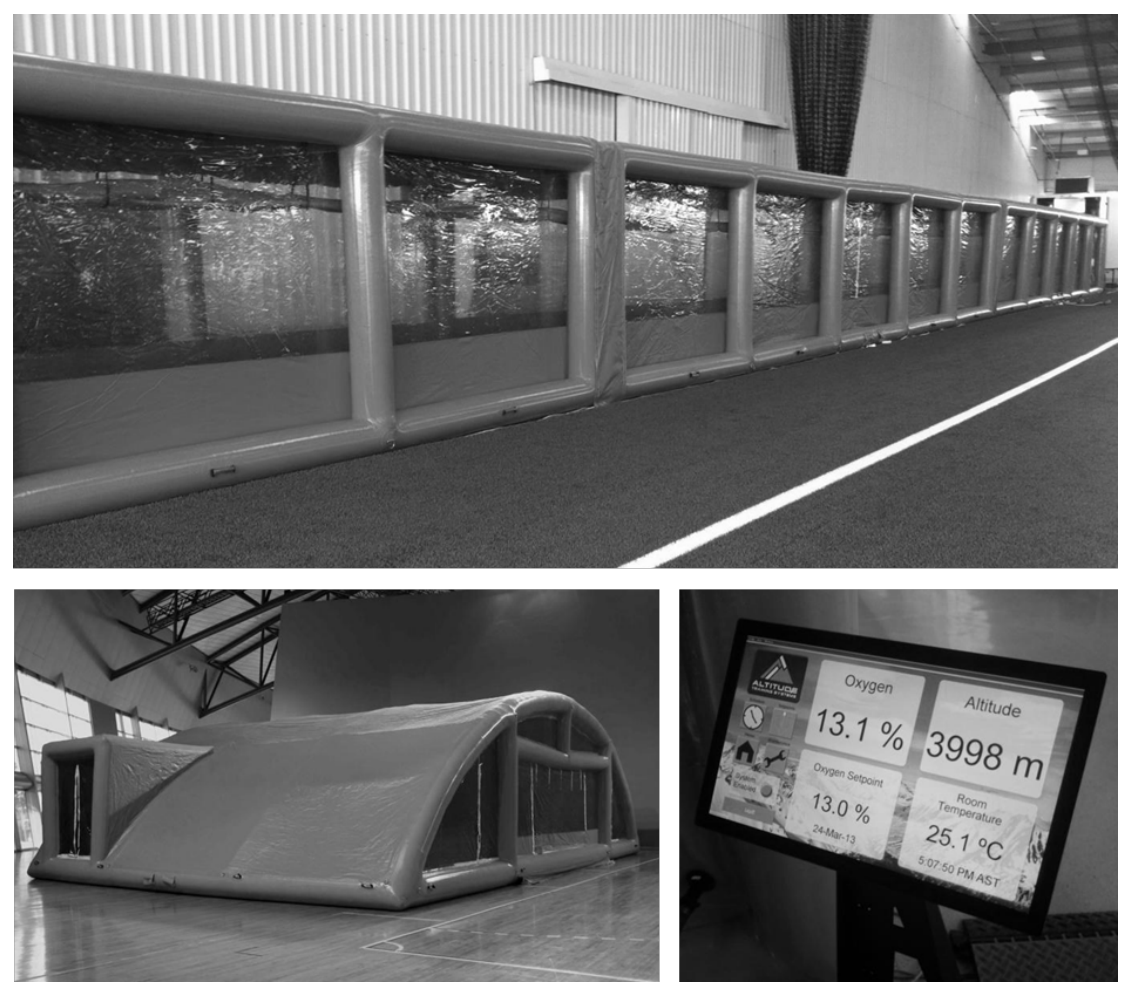

Figura 1. Presentación del sistema de hipoxia simulada inflable móvil. Túnel externo (panel superior), diseño rectangular (panel inferior izquierdo) y controlador de pantalla táctil (panel inferior derecho).

\section{Desafío a los métodos tradicionales en altitud}

Más allá de la innovación tecnológica, las diferencias intrínsecas entre los factores subyacentes de la resistencia (p. ej., velocidad máxima aeróbica, economía de carrera) y el rendimiento en deportes de equipo (p.ej. RSA) así como las limitaciones en los calendarios de competición explican por qué los objetivos y contenidos de los métodos hipóxicos y su periodización en el programa anual son en gran parte diferentes entre deportes individuales y de equipo. Para adaptarse a las nuevas limitaciones de los métodos hipóxicos contemporáneos ofrecidos a jugadores de deportes de equipo, la nomenclatura de Wilber fue recientemente modificada (Millet, y col., 2013, Millet, y col., 2010). En 2010, Millet y col. presentaron la posibilidad de combinar diferentes métodos hipóxicos. Los nuevos enfoques incluyen la "estancia intermitente hipóxica durante el entrenamiento de intervalo" (IHIT = IHT + IHE) y el "vivir arriba-entrenar abajo y arriba" (LHTLH = LHTL + IHT o RSH). Por ejemplo, con LHTLH donde atletas "viven en altitud y se entrenan al nivel del mar a excepción de unos intensos entrenamientos en altitud" (Millet, y col., 2010), los beneficios adicionales con respecto tanto a la capacidad aeróbica específica como a RSA han sido observados en jugadores de deportes de equipo (Brocherie, y col., 2015c). Con una "dosis hipóxica" baja $(\geq 200 \mathrm{~h}$ ), un entrenamiento LHTLH de 2 semanas realizado durante la temporada de jugadores de hockey sobre césped de nivel élite fue eficiente para obtener la regulación inmediata de la masa de hemoglobina $(+4 \%)$ y un aumento de rendimiento en el test Yo-Yo de recuperación intermitente de nivel $2(+20 \%)$ con incrementos similares en comparación con el entrenamiento al nivel del mar (LHTL + RSN) (Brocherie, y col., 2015c). Cabe destacar que la superioridad de LHTLH respecto a LHTL + RSN quedó demostrada en el rendimiento RSA con incrementos dos veces mayores que se mantienen al menos durante 3 semanas posteriores a LHTLH. Del mismo modo, la utilidad de la combinación de LHTH con entrenamiento de alta intensidad al nivel del mar ("vivir arriba-entrenar arriba y abajo"; LHTHL) también se demostró en nadadores (Rodriguez, Iglesias, Feriche, Calderon-Soto, 
Brocherie, F.; Girard, O.; Fais, R., y Millet, G. P. (2016). Altitud y deportes de equipo: métodos tradicionales desafiados por un entrenamiento innovador y específico en hipoxia. RICYDE. Revista internacional de ciencias del deporte. 46(12), 338-358. http://dx.doi.org/10.5232/ricyde2016.04601

Chaverri, Wachsmuth, Schmidt y Levine, 2015). Al disponer de más información sobre la intensificación de la glucólisis y la capacidad de amortiguación, hablamos también de los beneficios potenciales de estos métodos hipóxicos para el rendimiento anaeróbico. Frente a la falta de consenso sobre el nombre de los métodos [p.ej. IHT se refiere a veces como activo (IHT) o pasivo (IHE) formas (Bassovitch, 2010) a pesar de diferente bases fisiológicas (Millet, y col., 2010)] y basado en la presente revisión y el prometedor avance tecnológico, el método LLTH podría dividirse en cuatro subgrupos; p. ej., IHE, CHT (entrenamiento continuo $>30$ min a baja intensidad en hipoxia), IHT (entrenamiento de intervalo en hipoxia) y RSH (Figura 2). Millet y col. (2013) también señalan la naturaleza del estrés hipóxico dentro de los métodos LLTH. Además, el "entrenamiento de resistencia en hipoxia", fue recientemente propuesto con o sin oclusión vascular con el propósito de promover la hipertrofia y la producción de potencia, pero con beneficios poco claros en la literatura actualmente disponible (Scott, Slattery, Sculley y Dascombe, 2014). Con la creciente evidencia que sugiere que la hipoxia hipobárica induce diferentes adaptaciones fisiológicas respecto a la hipoxia normobárica (Millet, Faiss y Pialoux, 2012), es primordial informar sistemáticamente del método de reducción de la presión parcial de $\mathrm{O}_{2}$ ambiente. Evidencias sugieren que estas dos condiciones no se pueden usar de forma intercambiable (Coppel, Hennis, Gilbert-Kawai y Grocott, 2015, DiPasquale, Strangman, Harris y Muza, 2015, Fulco, Muza, Beidleman, Demes, Staab, Jones y Cymerman, 2011, Saugy, Schmitt, Cejuela, Faiss, Hauser, Wehrlin, Rudaz, Delessert, Robinson y Millet, 2014). Brevemente, la hipoxia hipobárica aumenta el espacio alveolar "muerto" asociado con alcalosis respiratoria e hipocapnia (Millet, y col., 2012), mejora el estrés oxidativo, combinado con una alteración de la biodisponibilidad del óxido nítrico (Faiss, Pialoux, Sartori, Faes, Deriaz y Millet, 2013b), y resulta en una acentuación del mal agudo de montaña (DiPasquale, y col., 2015), así como mayores trastornos respiratorios durante el sueño (Heinzer, Saugy, Rupp, Tobback, Faiss, Bourdillon, Rubio y Millet, 2016). Mientras que el coste energético de alta intensidad se reduce en hipoxia hipobárica frente a la hipoxia normobárica, y no afecta negativamente a la disponibilidad de energía (Peronnet, Thibault y Cousineau, 1991), la hipoxia hipobárica también resulta en respuestas de ventilación inferiores (Faiss, y col., 2013b) y una menor fatiga al nivel ventilatorio similar en comparación a la hipoxia normobárica. Así, con una mayor eficacia de la pre-aclimatación en hipoxia hipobárica (Fulco, Beidleman y Muza, 2013), la mejora del rendimiento al nivel del mar también puede ser diferente entre hipoxia hipobárica e hipoxia normobárica. Por ejemplo, la mayoría de los estudios LHTL hipobárica reportan mejoras del rendimiento $(+4.0 \% \mathrm{y}+4.2 \%$ de cambio en la producción de energía para los atletas de élite y no élite (Bonetti y Hopkins, 2009)) y/o hematológicas (Bonetti y Hopkins, 2009, Chapman, Karlsen, Resaland, Ge, Harber, Witkowski, Stray-Gundersen y Levine, 2014, Garvican-Lewis, Halliday, Abbiss, Saunders y Gore, 2015, Saugy, y col., 2014, Wehrlin y Marti, 2006). Por el contrario, los resultados positivos $(+0.6 \% \mathrm{y}+1.4 \%$ para los atletas de élite y no élite) han sido menos frecuentes en condiciones de hipoxia normobárica (Bonetti y Hopkins, 2009, Clark, Quod, Clark, Martin, Saunders y Gore, 2009, Robach, Schmitt, Brugniaux, Roels, Millet, Hellard, Nicolet, Duvallet, Fouillot, Moutereau, Lasne, Pialoux, Olsen y Richalet, 2006, Robertson, Saunders, Pyne, Aughey, Anson y Gore, 2010). Sin embargo, el conocimiento actual es insuficiente para confirmar si la hipoxia hipobárica o hipoxia normobárica induce una mayor mejora del rendimiento después de las intervenciones LHTL, mientras que el soporte científico es aún más frágil para los estudios RSH. Es difícil comparar los resultados entre los estudios con diferentes "dosis hipóxicas", cargas de entrenamiento, temperatura ambiente y con análisis estadísticos empleados (Coppel, y col., 2015, Millet, y col., 2012). Prácticamente, cada condición tiene ventajas y desventajas. En hipoxia hipobárica, se puede conducir una "dosis hipóxica" más larga durante un período de entrenamiento dado, pero las limitaciones logísticas relacionadas con el entrenamiento de 
Brocherie, F.; Girard, O.; Fais, R., y Millet, G. P. (2016). Altitud y deportes de equipo: métodos tradicionales desafiados por un entrenamiento innovador y específico en hipoxia. RICYDE. Revista internacional de ciencias del deporte. 46(12), 338-358. http://dx.doi.org/10.5232/ricyde2016.04601

deporte de equipo pueden ser perjudiciales. Al contrario, hipoxia normobárica permite individualizar el estímulo hipóxico. Además, mientras que una sesión única de ejercicio anaeróbico puede ejecutarse con la misma intensidad en hipoxia moderada o severa en comparación con normoxia (Álvarez-Herms, Julià-Sánchez, Gatterer, Blank, Corbi, Pagès, Burtscher y Viscor, 2016), este tipo de entrenamiento hipóxico puede ser útil para mejorar la respuesta psicofisiológica (p. ej. la tolerancia al esfuerzo percibido, la dificultad de respiración e la incomodidad en los miembros inferiores) a una "dosis hipóxica" específica y, en consecuencia ampliar el rendimiento físico y la tolerancia a la fatiga (Brocherie, Millet y Girard, 2016).

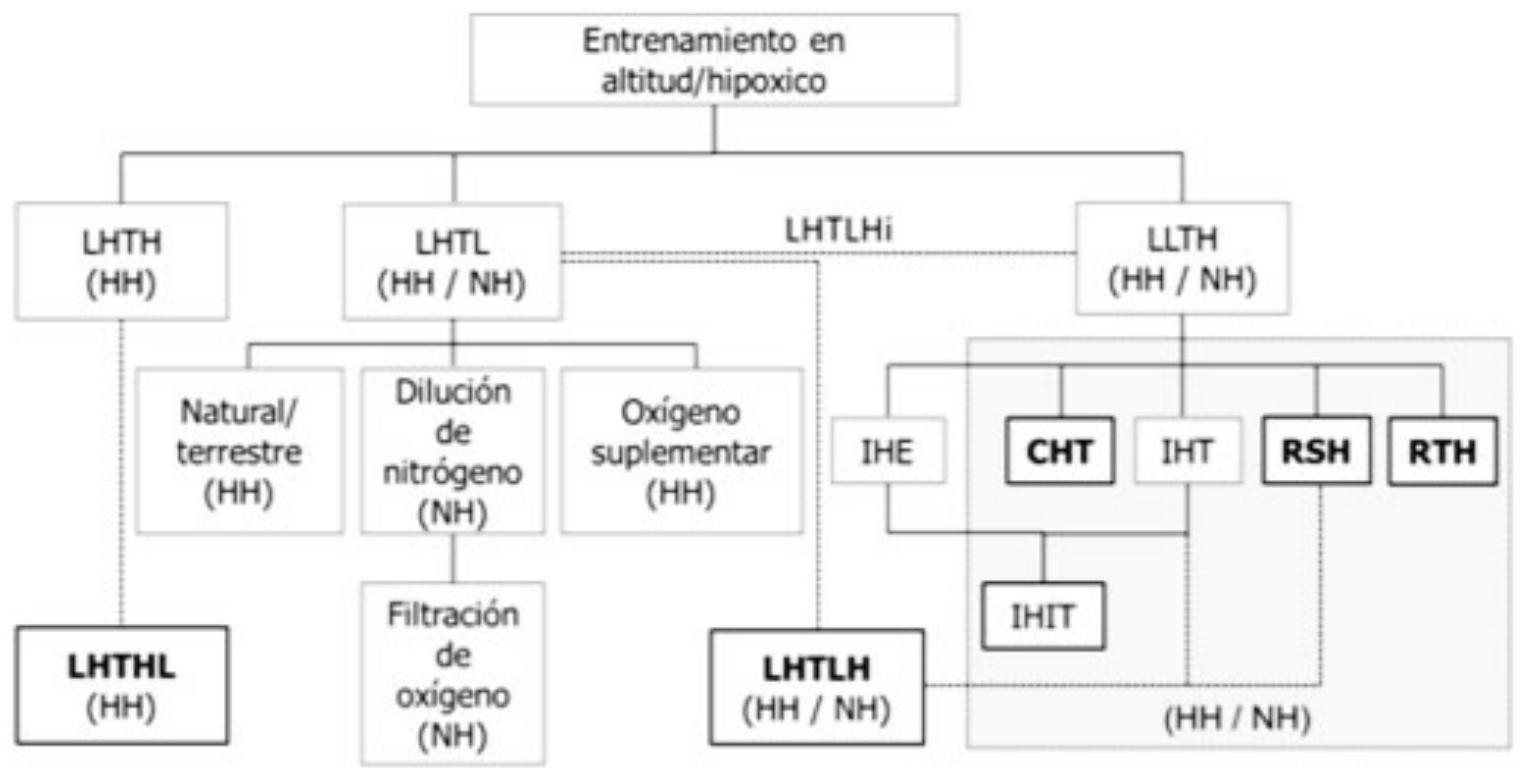

Figura 2. Panorama actualizado de los diferentes métodos hipóxicos disponibles para una amplia gama de atletas que participan en disciplinas de deporte de equipo y de resistencia. [(Millet, y col., 2013), adaptado de Wilber y col. (2007) y Millet y col. (2010) con cambios propuestos destacados en negrita]. LHTH: "vivir arriba-entrenar arriba"; LHTL: "vivir arriba-entrenar abajo"; LHTLH: "vivir arriba-entrenar abajo y arriba"; LHTHL: "vivir arriba-entrenar arriba y abajo"; LLTH: "vivir abajo-entrenar arriba"; LHTLHi: "vivir arriba-entrenar abajo y arriba", alternado con LLTH; IHE: "estancia intermitente hipóxica"; CHT: "entrenamiento continuo en hipoxia"; IHT: "entrenamiento hipóxico intermitente"; RSH: "entrenamiento de sprints repetidos en hipoxia"; RTH: "entrenamiento de resistencia en hipoxia",; IHIT: "estancia intermitente hipóxica durante el entrenamiento de intervalo"; NH: hipoxia normobárica; HH: hipoxia hipobárica.

\section{Conclusiones}

Un análisis exhaustivo de los estudios que han utilizado IHT conduce a beneficios sorprendentemente pobres para la mejora del rendimiento al nivel del mar, en comparación con el mismo protocolo de entrenamiento realizado en normoxia. A pesar de observarse adaptaciones moleculares positivas después de varias modalidades de IHT, las características del estímulo del entrenamiento óptimo en hipoxia son todavía confusas y su traducción funcional en términos de mejora del rendimiento del cuerpo entero es mínima. Para superar algunas de las limitaciones inherentes del IHT (p. ej., menor estimulo de entrenamiento debido a la hipoxia), estudios recientes han investigado un nuevo método de entrenamiento llamado RSH que se basa en la repetición de sprints en hipoxia. La sucesión de esfuerzos máximos en condiciones de hipoxia ha demostrado ser beneficioso para la mejora del rendimiento máximo y, especialmente, para retrasar el cansancio cuando los sprints con recuperaciones incompletas se repiten hasta el agotamiento. 
Brocherie, F.; Girard, O.; Fais, R., y Millet, G. P. (2016). Altitud y deportes de equipo: métodos tradicionales desafiados por un entrenamiento innovador y específico en hipoxia. RICYDE. Revista internacional de ciencias del deporte. 46(12), 338-358. http://dx.doi.org/10.5232/ricyde2016.04601

Por lo tanto, RSH se propone como una estrategia de entrenamiento prometedora para mejorar el rendimiento físico en deportes intermitentes. Dado que los deportes de equipo se caracterizan por los episodios de ejercicio intenso repetidos a lo largo de un partido, retrasar el cansancio y mejorar la capacidad de repetición de sprints es crucial para mejorar la participación física de los jugadores. Hasta ahora, hay escasa prueba sobre los beneficios adicionales en RSA después un entrenamiento de alta intensidad realizado en hipoxia en comparación con el mismo entrenamiento en normoxia. Es también prematuro evaluar si los mecanismos sugeridos (vasodilatación y efectos potenciales de fibra selectiva) para sustentar el mejor rendimiento inducido por RSH son similares o no a aquellos derivados de los estudios de suplementación de nitrato. Sin embargo, según nuestra opinión, éstos explican parcialmente la diferencia de eficacia entre los métodos RSH e IHT de cara a mejorar el rendimiento locomotor. Hasta que no se proporcionen nuevas pruebas, se considera que RSH se basa en diferentes mecanismos fundamentales que podrían depender del tipo de fibra, mientras que las adaptaciones positivas son probablemente dependientes de los efectos vasodilatadores compensatorios sobre el comportamiento de fibras FT.

Se necesitan más estudios con protocolos diseñados en doble ciego y con muestra de gran tamaño para respaldar la eficacia del RSH. La valoración del impacto del RSH sobre el rendimiento físico en varios deportes de equipo podría mejorarse significativamente comprobando, p. ej., determinados ratios de ejercicio-descanso o la eficacia de diferentes "dosis hipóxicas". Por último, si la eficacia del RSH se confirma en situaciones más ecológicas (p.ej. mediante el uso de la innovación tecnológica como el sistema de hipoxia simulada), podría ser fácilmente implementado en la periodización anual de disciplinas intermitentes. Al ajustarse a las necesidades específicas de los jugadores de deportes de equipo, el uso de este tipo de equipamiento abrirá nuevas fronteras para optimizar las futuras aplicaciones del entrenamiento hipóxico (p.ej., pre-aclimatación, rehabilitación, mantenimiento de rendimiento, eficacia de métodos hipóxicos hipobáricos frente a métodos hipóxicos normobáricos).

\section{Agradecimientos}

Los autores agradecen a Aspetar (Qatar Orthopaedic and Sports Medicine Hospital) la financiación del equipo de hipoxia simulada inflable móvil aquí descrito. No se ha recurrido a ninguna fuente de financiación para ayudar en la preparación de este artículo. Los autores no tienen conflictos de intereses que sean directamente relevantes para el contenido de este artículo.

\section{Referencias}

Álvarez-Herms J.; Julià-Sánchez S.; Gatterer H.; Blank C.; Corbi F.; Pagès T.; Burtscher M., \& Viscor G. (2016) Anaerobic training in hypoxia: A new approach to stimulate the rating of effort perception. Physiology and Behavior, 163, 37-42.

http://dx.doi.org/10.1016/j.physbeh.2016.04.035

Aughey, R. J.; Clark, S. A.; Gore, C. J., Townsend, N. E.; Hahn, A. G.; Kinsman, T. A.; Goodman, C.; Chow, C. M.; Martin, D. T.; Hawley, J. A., \& McKenna, M. J. (2006) Interspersed normoxia during live high, train low interventions reverses an early reduction in muscle $\mathrm{Na}+, \mathrm{K}+$ ATPase activity in well-trained athletes. European Journal of Applied Physiology \& Occupational Physiology, 98, 299-309.

http://dx.doi.org/10.1007/s00421-006-0280-z

Balsom, P. D.; Gaitanos, G. C.; Ekblom, B., \& Sjodin, B. (1994) Reduced oxygen availability during high intensity intermittent exercise impairs performance. Acta Physiologica Scandinavica, 152, 279-285.

http://dx.doi.org/10.1111/j.1748-1716.1994.tb09807.x 
Brocherie, F.; Girard, O.; Fais, R., y Millet, G. P. (2016). Altitud y deportes de equipo: métodos tradicionales desafiados por un entrenamiento innovador y específico en hipoxia. RICYDE. Revista internacional de ciencias del deporte. 46(12), 338-358. http://dx.doi.org/10.5232/ricyde2016.04601

Bartsch, P.; Dvorak, J., \& Saltin, B. (2008) Football at high altitude. Scandinavian Journal of Medicine and Science in Sports, 18, iii-iv.

http://dx.doi.org/10.1111/j.1600-0838.2008.00826.x

Bartsch, P.; Dvorak, J., \& Saltin, B. (2009) A rebuttal. Scandinavian Journal of Medicine and Science in Sports, 19, 608.

http://dx.doi.org/10.1111/j.1600-0838.2009.00994.x

Bartsch, P.; Saltin, B., \& Dvorak, J. (2008) Consensus statement on playing football at different altitude. Scandinavian Journal of Medicine and Science in Sports, 18 Suppl 1, 96-99. http://dx.doi.org/10.1111/j.1600-0838.2008.00837.x

Bassovitch, O. (2010) 'Combining hypoxic methods for peak performance': a biomedical engineering perspective. Sports Medicine, 40, 519-521; author reply 521-513. http://dx.doi.org/10.2165/11535150-000000000-00000

Bergeron, M. F.; Bahr, R.; Bartsch, P.; Bourdon, L.; Calbet, J. A.; Carlsen, K. H.; Castagna, O.; Gonzalez-Alonso, J.; Lundby, C.; Maughan, R. J.; Millet, G.; Mountjoy, M.; Racinais, S.; Rasmussen, P.; Singh, D. G.; Subudhi, A. W.; Young, A. J.; Soligard, T., \& Engebretsen, L. (2012) International Olympic Committee consensus statement on thermoregulatory and altitude challenges for high-level athletes. British Journal of Sports Medicine, 46, 770-779.

http://dx.doi.org/10.1136/bjsports-2012-091296

Billaut, F.; Gore, C. J., \& Aughey, R. J. (2012) Enhancing team-sport athlete performance: is altitude training relevant? Sports Medicine, 42, 751-767.

http://dx.doi.org/10.1007/BF03262293

Bonetti, D. L., \& Hopkins, W. G. (2009) Sea-level exercise performance following adaptation to hypoxia: a meta-analysis. Sports Medicine, 39, 107-127. http://dx.doi.org/10.2165/00007256-200939020-00002

Brocherie, F.; Girard, O.; Faiss, R., \& Millet, G. P. (2015a) High-intensity intermittent training in hypoxia: a double-blinded, placebo-controlled field study in youth football players. Journal of Strength and Conditioning Research, 29, 226-237. http://dx.doi.org/10.1519/JSC.0000000000000590

Brocherie, F.; Millet, G. P., \& Girard, O. (2015b) Neuro-mechanical and metabolic adjustments to the repeated anaerobic sprint test in professional football players. European Journal of Applied Physiology, 115, 891-903. http://dx.doi.org/10.1007/s00421-014-3070-z

Brocherie, F.; Millet, G. P.; Hauser, A.; Steiner, T.; Rysman, J.; Wehrlin, J. P., \& Girard, O. (2015c) "Live High-Train Low and High" Hypoxic Training Improves Team-Sport Performance. Medicine and Science in Sports and Exercise, 47, 2140-2149. http://dx.doi.org/10.1249/MSS.0000000000000630

Brocherie F.; Millet GP., \& Girard O. (2016) Psycho-Physiological Responses to RepeatedSprint Training in Normobaric Hypoxia and Normoxia. International Journal of Sports Physiology and Performance, [Epub ahead of print].

http://dx.doi.org/10.1123/ijspp.2016-0052

Buchheit, M.; Kuitunen, S.; Voss, S. C.; Williams, B. K.; Mendez-Villanueva, A., \& Bourdon, P. C. (2012) Physiological strain associated with high-intensity hypoxic intervals in highly trained young runners. Journal of Strength and Conditioning Research, 26, 94-105. http://dx.doi.org/10.1519/JSC.0b013e3182184fcb

Casey, D. P., \& Joyner, M. J. (2012) Compensatory vasodilatation during hypoxic exercise: mechanisms responsible for matching oxygen supply to demand. Journal of Physiology, 590, 6321-6326.

http://dx.doi.org/10.1113/jphysiol.2012.242396 
Brocherie, F.; Girard, O.; Fais, R., y Millet, G. P. (2016). Altitud y deportes de equipo: métodos tradicionales desafiados por un entrenamiento innovador y específico en hipoxia. RICYDE. Revista internacional de ciencias del deporte. 46(12), 338-358. http://dx.doi.org/10.5232/ricyde2016.04601

Chapman, R. F.; Karlsen, T.; Resaland, G. K.; Ge, R. L.; Harber, M. P.; Witkowski, S.; Stray-Gundersen, J., \& Levine, B. D. (2014) Defining the "dose" of altitude training: how high to live for optimal sea level performance enhancement. Journal of Applied Physiology (1985), 116, 595-603.

http://dx.doi.org/10.1152/japplphysiol.00634.2013

Clark, S. A.; Quod, M. J.; Clark, M. A.; Martin, D. T.; Saunders, P. U., \& Gore, C. J. (2009) Time course of haemoglobin mass during 21 days live high:train low simulated altitude. European Journal of Applied Physiology, 106, 399-406.

http://dx.doi.org/10.1007/s00421-009-1027-4

Cleland, S. M.; Murias, J. M.; Kowalchuk, J. M., \& Paterson, D. H. (2012) Effects of prior heavy-intensity exercise on oxygen uptake and muscle deoxygenation kinetics of a subsequent heavy-intensity cycling and knee-extension exercise. Applied Physiology, Nutrition and Metabolism, 37, 138-148.

http://dx.doi.org/10.1139/h11-143

Coppel, J.; Hennis, P.; Gilbert-Kawai, E., \& Grocott, M. P. (2015) The physiological effects of hypobaric hypoxia versus normobaric hypoxia: a systematic review of crossover trials. Extreme Physiology and Medicine, 4, 2.

http://dx.doi.org/10.1186/s13728-014-0021-6

Crowley, W. F., Jr. (2003) Translation of basic research into useful treatments: how often does it occur? The American Journal of Medicine, 114, 503-505.

http://dx.doi.org/10.1016/S0002-9343(03)00119-0

Daniels, J., \& Oldridge, N. (1970) The effects of alternate exposure to altitude and sea level on world-class middle-distance runners. Medicine and Science in Sports, 2, 107112. http://dx.doi.org/10.1249/00005768-197023000-00001

DiPasquale, D. M.; Strangman, G. E.; Harris, N. S., \& Muza, S. R. (2015) Hypoxia, Hypobaria, and Exercise Duration Affect Acute Mountain Sickness. Aerospace Medicine and Human Performance, 86, 614-619.

http://dx.doi.org/10.3357/AMHP.4266.2015

Dufour, S. P.; Ponsot, E.; Zoll, J.; Doutreleau, S.; Lonsdorfer-Wolf, E.; Geny, B.; Lampert, E.; Fluck, M.; Hoppeler, H.; Billat, V.; Mettauer, B.; Richard, R., \& Lonsdorfer, J. (2006) Exercise training in normobaric hypoxia in endurance runners. I. Improvement in aerobic performance capacity. Journal of Applied Physiology, 100, 1238-1248.

http://dx.doi.org/10.1152/japplphysiol.00742.2005

Faiss, R.; Leger, B.; Vesin, J. M.; Fournier, P. E.; Eggel, Y.; Deriaz, O., \& Millet, G. P. (2013a) Significant molecular and systemic adaptations after repeated sprint training in hypoxia. PLoS One, 8, e56522.

http://dx.doi.org/10.1371/journal.pone.0056522

Faiss, R.; Pialoux, V.; Sartori, C.; Faes, C.; Deriaz, O., \& Millet, G. P. (2013b) Ventilation, Oxidative Stress and Nitric Oxide in Hypobaric vs. Normobaric Hypoxia. Medicine and Science in Sports and Exercise, 45, 253-260.

http://dx.doi.org/10.1249/MSS.0b013e31826d5aa2

Faiss, R.; Willis, S.; Born, D. P.; Sperlich, B.; Vesin, J. M.; Holmberg, H. C., \& Millet, G. P. (2015) Repeated double-poling sprint training in hypoxia by competitive crosscountry skiers. Medicine and Science in Sports and Exercise, 47, 809-817. http://dx.doi.org/10.1249/MSS.0000000000000464

Friedmann, B.; Frese, F.; Menold, E.; Kauper, F.; Jost, J., \& Bartsch, P. (2005) Individual variation in the erythropoietic response to altitude training in elite junior swimmers. British Journal of Sports Medicine, 39, 148-153.

http://dx.doi.org/10.1136/bjsm.2003.011387 
Brocherie, F.; Girard, O.; Fais, R., y Millet, G. P. (2016). Altitud y deportes de equipo: métodos tradicionales desafiados por un entrenamiento innovador y específico en hipoxia. RICYDE. Revista internacional de ciencias del deporte. 46(12), 338-358. http://dx.doi.org/10.5232/ricyde2016.04601

Fulco, C. S.; Beidleman, B. A., \& Muza, S. R. (2013) Effectiveness of preacclimatization strategies for high-altitude exposure. Exercise and Sport Sciences Reviews, 41, 55-63. http://dx.doi.org/10.1097/JES.0b013e31825eaa33

Fulco, C. S.; Muza, S. R.; Beidleman, B. A.; Demes, R., Staab, J. E.; Jones, J. E., \& Cymerman, A. (2011) Effect of repeated normobaric hypoxia exposures during sleep on acute mountain sickness, exercise performance, and sleep during exposure to terrestrial altitude. The American Journal of Physiology - Regulatory, Integrative and Comparative Physiology, 300, R428-436.

http://dx.doi.org/10.1152/ajpregu.00633.2010

Galvin, H. M.; Cooke, K.; Sumners, D. P.; Mileva, K. N., \& Bowtell, J. L. (2013) Repeated sprint training in normobaric hypoxia. British Journal of Sports Medicine, 47 Suppl 1, i74-i79.

http://dx.doi.org/10.1136/bjsports-2013-092826

Garvican-Lewis, L. A.; Halliday, I.; Abbiss, C. R.; Saunders, P. U., \& Gore, C. J. (2015) Altitude Exposure at $1800 \mathrm{~m}$ Increases Haemoglobin Mass in Distance Runners. Journal of Sports Science and Medicine, 14, 413-417.

Gatterer, H.; Faulhaber, M., \& Netzer, N. (2009) Hypoxic training for football players. Scandinavian Journal of Medicine and Science in Sports, 19, 607; author reply 608. http://dx.doi.org/10.1111/j.1600-0838.2009.00993.x

Gatterer, H.; Philippe, M.; Menz, V.; Mosbach, F.; Faulhaber, M., \& Burtscher, M. (2014) Shuttle-run sprint training in hypoxia for youth elite soccer players: a pilot study. Journal of Sports Science and Medicine, 13, 731-735.

Girard, O.; Amann, M.; Aughey, R.; Billaut, F.; Bishop, D. J.; Bourdon, P.; Buchheit, M.; Chapman, R.; D'Hooghe, M.; Garvican-Lewis, L. A.; Gore, C. J.; Millet, G. P.; Roach, G. D.; Sargent, C.; Saunders, P. U.; Schmidt, W., \& Schumacher, Y. O. (2013a) Position statement--altitude training for improving team-sport players' performance: current knowledge and unresolved issues. British Journal of Sports Medicine, 47 Suppl 1 , i8-16.

http://dx.doi.org/10.1136/bjsports-2013-093109

Girard, O.; Brocherie, F., \& Millet, G. P. (2013b) On the use of mobile inflatable hypoxic marquees for sport-specific altitude training in team sports. British Journal of Sports Medicine, 47 Suppl 1, i121-i123.

http://dx.doi.org/10.1136/bjsports-2013-092794

Girard, O.; Racinais, S.; Kelly, L.; Millet, G. P., \& Brocherie, F. (2011) Repeated sprinting on natural grass impairs vertical stiffness but does not alter plantar loading in soccer players. European Journal of Applied Physiology, 111, 2547-2555.

http://dx.doi.org/10.1007/s00421-011-1884-5

Gore, C. J.; Hahn, A.; Rice, A.; Bourdon, P.; Lawrence, S.; Walsh, C.; Stanef, T.; Barnes, P.; Parisotto, R.; Martin, D., \& Pyne, D. (1998) Altitude training at $2690 \mathrm{~m}$ does not increase total haemoglobin mass or sea level VO2max in world champion track cyclists. Journal of Science and Medicine in Sport, 1, 156-170.

http://dx.doi.org/10.1016/S1440-2440(98)80011-X

Gore, C. J.; Hahn, A. G.; Aughey, R. J.; Martin, D. T.; Ashenden, M. J.; Clark, S. A.; Garnham, A. P.; Roberts, A. D.; Slater, G. J., \& McKenna, M. J. (2001) Live high:train low increases muscle buffer capacity and submaximal cycling efficiency. Acta Physiologica Scandinavica, 173, 275-286.

http://dx.doi.org/10.1152/japplphysiol.00342.2006 
Brocherie, F.; Girard, O.; Fais, R., y Millet, G. P. (2016). Altitud y deportes de equipo: métodos tradicionales desafiados por un entrenamiento innovador y específico en hipoxia. RICYDE. Revista internacional de ciencias del deporte. 46(12), 338-358. http://dx.doi.org/10.5232/ricyde2016.04601

Gore, C. J.; Rodriguez, F. A.; Truijens, M. J.; Townsend, N. E.; Stray-Gundersen, J., \& Levine, B. D. (2006) Increased serum erythropoietin but not red cell production after 4 wk of intermittent hypobaric hypoxia (4,000-5,500 m). Journal of Applied Physiology, $101,1386-1393$.

http://dx.doi.org/10.1046/j.1365-201X.2001.00906.x

Hamlin, M. J.; Hinckson, E. A.; Wood, M. R., \& Hopkins, W. G. (2008) Simulated rugby performance at $1550-\mathrm{m}$ altitude following adaptation to intermittent normobaric hypoxia. Journal of Science and Medicine in Sport, 11, 593-599. http://dx.doi.org/10.1016/j.jsams.2007.07.005

Hamlin, M. J.; Marshall, H. C.; Hellemans, J.; Ainslie, P. N., \& Anglem, N. (2010a) Effect of intermittent hypoxic training on $20 \mathrm{~km}$ time trial and $30 \mathrm{~s}$ anaerobic performance. Scandinavian Journal of Medicine and Science in Sports, 20, 651-661. http://dx.doi.org/10.1111/j.1600-0838.2009.00946.x

Hamlin, M. J.; Marshall, H. C.; Hellemans, J.; Ainslie, P. N., \& Anglem, N. (2010b) Effect of intermittent hypoxic training on $20 \mathrm{~km}$ time trial and $30 \mathrm{~s}$ anaerobic performance. Scandinavian Journal of Medicine and Science in Sports, 20, 651-661. http://dx.doi.org/10.1111/j.1600-0838.2009.00946.x

Heinzer, R.; Saugy, J. J.; Rupp, T.; Tobback, N.; Faiss, R.; Bourdillon, N.; Rubio, J. H., \& Millet, G. P. (2016) Comparison of Sleep Disorders between Real and Simulated 3,450-m Altitude. Sleep. [Epub ahead of print].

Hendriksen, I. J., \& Meeuwsen, T. (2003) The effect of intermittent training in hypobaric hypoxia on sea-level exercise: a cross-over study in humans. European Journal of Applied Physiology \& Occupational Physiology, 88, 396-403. http://dx.doi.org/10.1007/s00421-002-0708-z

Holliss, B. A.; Fulford, J.; Vanhatalo, A.; Pedlar, C. R., \& Jones, A. M. (2013) Influence of intermittent hypoxic training on muscle energetics and exercise tolerance. Journal of Applied Physiology, 114, 611-619.

http://dx.doi.org/10.1152/japplphysiol.01331.2012

Hoppeler, H., \& Vogt, M. (2001) Muscle tissue adaptations to hypoxia. Journal of Experimental Biology, 204, 3133-3139.

Iaia, F. M.; Rampinini, E., \& Bangsbo, J. (2009) High-intensity training in football. International Journal of Sports Physiology and Performance, 4, 291-306.

Kasai, N.; Mizuno, S.; Ishimoto, S.; Sakamoto, E.; Maruta, M., \& Goto, K. (2015) Effect of training in hypoxia on repeated sprint performance in female athletes. Springerplus, $4,310$.

http://dx.doi.org/10.1186/s40064-015-1041-4

Katayama, K.; Sato, Y.; Morotome, Y.; Shima, N.; Ishida, K.; Mori, S., \& Miyamura, M. (1999) Ventilatory chemosensitive adaptations to intermittent hypoxic exposure with endurance training and detraining. Journal of Applied Physiology, 86, 1805-1811.

Kime, R.; Karlsen, T.; Nioka, S.; Lech, G.; Madsen, O.; Saeterdal, R.; Im, J.; Chance, B., \& Stray-Gundersen, J. (2003) Discrepancy between cardiorespiratory system and skeletal muscle in elite cyclists after hypoxic training. Dynamic Medicine, 2, 4. http://dx.doi.org/10.1186/1476-5918-2-4

Klein, C. S.; Marsh, G. D.; Petrella, R. J., \& Rice, C. L. (2003) Muscle fiber number in the biceps brachii muscle of young and old men. Muscle Nerve, 28, 62-68. http://dx.doi.org/10.1002/mus.10386

Levine, B. D., \& Stray-Gundersen, J. (2006) Dose-response of altitude training: how much altitude is enough? Advances in Experimental Medicine and Biology, 588, 233247. http://dx.doi.org/10.1007/978-0-387-34817-9_20 
Brocherie, F.; Girard, O.; Fais, R., y Millet, G. P. (2016). Altitud y deportes de equipo: métodos tradicionales desafiados por un entrenamiento innovador y específico en hipoxia. RICYDE. Revista internacional de ciencias del deporte. 46(12), 338-358. http://dx.doi.org/10.5232/ricyde2016.04601

Lundby, C.; Calbet, J. A., \& Robach, P. (2009) The response of human skeletal muscle tissue to hypoxia. Cellular and Molecular Life Sciences, 66, 3615-3623. http://dx.doi.org/10.1007/s00018-009-0146-8

Lundby, C.; Millet, G. P.; Calbet, J. A.; Bartsch, P., \& Subudhi, A. W. (2012) Does 'altitude training' increase exercise performance in elite athletes? British Journal of Sports Medicine, 46, 792-795.

http://dx.doi.org/10.1136/bjsports-2012-091231

Manimmanakorn, A.; Hamlin, M. J.; Ross, J. J.; Taylor, R., \& Manimmanakorn, N. (2012) Effects of low-load resistance training combined with blood flow restriction or hypoxia on muscle function and performance in netball athletes. Journal of Science and Medicine in Sport, 16, 337-342.

http://dx.doi.org/10.1016/j.jsams.2012.08.009

Manimmanakorn, A.; Manimmanakorn, N.; Taylor, R.; Draper, N.; Billaut, F.; Shearman, J. P., \& Hamlin, M. J. (2013) Effects of resistance training combined with vascular occlusion or hypoxia on neuromuscular function in athletes. European Journal of Applied Physiology, 113, 1767-1774.

http://dx.doi.org/10.1007/s00421-013-2605-z

Martino, M.; Myers, K., \& Bishop, P. (1995) Effects of 21 days training at altitude on sealevel anaerobic performance in competitive swimmers. Medicine and Science in Sports and Exercise, 27, (abstract 37).

http://dx.doi.org/10.1249/00005768-199505001-00037

McDonough, P.; Behnke, B. J.; Padilla, D. J.; Musch, T. I., \& Poole, D. C. (2005) Control of microvascular oxygen pressures in rat muscles comprised of different fibre types. Journal of Physiology, 563, 903-913.

http://dx.doi.org/10.1113/jphysiol.2004.079533

Mendez-Villanueva, A.; Edge, J.; Suriano, R.; Hamer, P., \& Bishop, D. (2012) The recovery of repeated-sprint exercise is associated with $\mathrm{PCr}$ resynthesis, while muscle $\mathrm{pH}$ and EMG amplitude remain depressed. PLOS ONE, 7, e51977.

http://dx.doi.org/10.1371/journal.pone.0051977

Millet, G. P., \& Faiss, R. (2012) Hypoxic conditions and exercise-to-rest ratio are likely paramount. Sports Medicine, 42, 1081-1083; author reply 1083-1085. http://dx.doi.org/ 10.2165/11640210-000000000-00000

Millet, G. P.; Faiss, R.; Brocherie, F., \& Girard, O. (2013) Hypoxic training and team sports: a challenge to traditional methods? British Journal of Sports Medicine, 47 Suppl 1, i6-i7.

http://dx.doi.org/10.1136/bjsports-2013-092793

Millet, G. P.; Faiss, R., \& Pialoux, V. (2012) Point: Hypobaric hypoxia induces different physiological responses from normobaric hypoxia. Journal of Applied Physiology, 112, 1783-1784.

http://dx.doi.org/10.1152/japplphysiol.00067.2012

Millet, G. P.; Roels, B.; Schmitt, L.; Woorons, X., \& Richalet, J. P. (2010) Combining hypoxic methods for peak performance. Sports Medicine, 40, 1-25.

http://dx.doi.org/10.2165/11317920-000000000-00000

Mizuno, M.; Juel, C.; Bro-Rasmussen, T.; Mygind, E.; Schibye, B.; Rasmussen, B., \& Saltin, B. (1990) Limb skeletal muscle adaptation in athletes after training at altitude. Journal of Applied Physiology, 68, 496-502.

Morton, J. P., \& Cable, N. T. (2005) Effects of intermittent hypoxic training on aerobic and anaerobic performance. Ergonomics, 48, 1535-1546.

http://dx.doi.org/10.1080/00140130500100959 
Brocherie, F.; Girard, O.; Fais, R., y Millet, G. P. (2016). Altitud y deportes de equipo: métodos tradicionales desafiados por un entrenamiento innovador y específico en hipoxia. RICYDE. Revista internacional de ciencias del deporte. 46(12), 338-358. http://dx.doi.org/10.5232/ricyde2016.04601

Peronnet, F.; Thibault, G., \& Cousineau, D. L. (1991) A theoretical analysis of the effect of altitude on running performance. Journal of Applied Physiology (1985), 70, 399404.

Ponsot, E.; Dufour, S. P.; Zoll, J.; Doutrelau, S.; N'Guessan, B.; Geny, B.; Hoppeler, H.; Lampert, E.; Mettauer, B.; Ventura-Clapier, R., \& Richard, R. (2006) Exercise training in normobaric hypoxia in endurance runners. II. Improvement of mitochondrial properties in skeletal muscle. Journal of Applied Physiology, 100, 1249-1257. http://dx.doi.org/10.1152/japplphysiol.00361.2005

Puype, J.; Van Proeyen, K.; Raymackers, J. M.; Deldicque, L., \& Hespel, P. (2013) Sprint Interval Training in Hypoxia Stimulates Glycolytic Enzyme Activity. Medicine and Science in Sports and Exercise, 45, 2166-2174. http://dx.doi.org/10.1249/MSS.0b013e31829734ae

Robach, P.; Schmitt, L.; Brugniaux, J. V.; Roels, B.; Millet, G.; Hellard, P.; Nicolet, G.; Duvallet, A.; Fouillot, J. P.; Moutereau, S.; Lasne, F.; Pialoux, V.; Olsen, N. V., \& Richalet, J. P. (2006) Living high-training low: effect on erythropoiesis and aerobic performance in highly-trained swimmers. European Journal of Applied Physiology, 96, 423-433.

http://dx.doi.org/10.1007/s00421-005-0089-1

Robertson, E. Y.; Saunders, P. U.; Pyne, D. B.; Aughey, R. J.; Anson, J. M., \& Gore, C. J. (2010) Reproducibility of performance changes to simulated live high/train low altitude. Medicine and Science in Sports and Exercise, 42, 394-401. http://dx.doi.org/10.1249/MSS.0b013e3181b34b57

Rodriguez, F. A.; Iglesias, X.; Feriche, B.; Calderon-Soto, C.; Chaverri, D.; Wachsmuth, N. B.; Schmidt, W., \& Levine, B. D. (2015) Altitude Training in Elite Swimmers for Sea Level Performance (Altitude Project). Medicine and Science in Sports and Exercise, 47, 1965-1978.

http://dx.doi.org/10.1249/MSS.0000000000000626

Roels, B.; Bentley, D. J.; Coste, O.; Mercier, J., \& Millet, G. P. (2007) Effects of intermittent hypoxic training on cycling performance in well-trained athletes. European Journal of Applied Physiology and Occupational Physiology, 101, 359-368.

http://dx.doi.org/10.1007/s00421-007-0506-8

Roels, B.; Millet, G. P.; Marcoux, C. J.; Coste, O.; Bentley, D. J., \& Candau, R. B. (2005) Effects of hypoxic interval training on cycling performance. Medicine and Science in Sports and Exercise, 37, 138-146.

http://dx.doi.org/10.1249/01.MSS.0000150077.30672.88

Roels, B.; Thomas, C.; Bentley, D. J.; Mercier, J.; Hayot, M., \& Millet, G. (2007) Effects of intermittent hypoxic training on amino and fatty acid oxidative combustion in human permeabilized muscle fibers. Journal of Applied Physiology, 102, 79-86. http://dx.doi.org/10.1152/japplphysiol.01319.2005

Roskamm, H.; Landry, F.; Samek, L.; Schlager, M.; Weidermann, H., \& Reindell, H. (1969) Effects of a standardized ergometer training program at three different altitudes. Journal of Applied Physiology, 27, 840-847.

Sanchis-Moysi, J.; Idoate, F.; Olmedillas, H.; Guadalupe-Grau, A.; Alayon, S.; Carreras, A.; Dorado, C., \& Calbet, J. A. (2010) The upper extremity of the professional tennis player: muscle volumes, fiber-type distribution and muscle strength. Scandinavian Journal of Medicine and Science in Sports, 20, 524-534. http://dx.doi.org/10.1111/j.1600-0838.2009.00969.x

Saugy, J. J.; Schmitt, L.; Cejuela, R.; Faiss, R.; Hauser, A.; Wehrlin, J. P.; Rudaz, B.; Delessert, A.; Robinson, N., \& Millet, G. P. (2014) Comparison of "Live High-Train Low" in normobaric versus hypobaric hypoxia. PLoS One, 9, e114418.

http://dx.doi.org/10.1371/journal. pone.0114418 
Brocherie, F.; Girard, O.; Fais, R., y Millet, G. P. (2016). Altitud y deportes de equipo: métodos tradicionales desafiados por un entrenamiento innovador y específico en hipoxia. RICYDE. Revista internacional de ciencias del deporte. 46(12), 338-358. http://dx.doi.org/10.5232/ricyde2016.04601

Scott, B. R.; Slattery, K. M.; Sculley, D. V., \& Dascombe, B. J. (2014) Hypoxia and Resistance Exercise: A Comparison of Localized and Systemic Methods. Sports Medicine, 44, 1037-1054. http://dx.doi.org/10.1007/s40279-014-0177-7

Semenza, G.L.; Shimoda, L. A., \& Prabhakar, N. R. (2006) Regulation of gene expression by HIF-1. Novartis Foundation Symposia, 272, 2-8; discussion 8-14, 33-16. http://dx.doi.org/10.1002/9780470035009.ch2

Smith, K. J., \& Billaut, F. (2010) Influence of cerebral and muscle oxygenation on repeated-sprint ability. European Journal of Applied Physiology, 109, 989-999. http://dx.doi.org/10.1007/s00421-010-1444-4

Toffoli, S.; Roegiers, A.; Feron, O.; Van Steenbrugge, M.; Ninane, N.; Raes, M., \& Michiels, C. (2009) Intermittent hypoxia is an angiogenic inducer for endothelial cells: role of HIF-1. Angiogenesis, 12, 47-67. http://dx.doi.org/10.1007/s10456-009-9131-y

Truijens, M. J.; Toussaint, H. M.; Dow, J., \& Levine, B. D. (2003) Effect of high-intensity hypoxic training on sea-level swimming performances. Journal of Applied Physiology, 94, 733-743. http://dx.doi.org/10.1152/japplphysiol.00079.2002

Vogt, M.; Puntschart, A.; Geiser, J.; Zuleger, C.; Billeter, R., \& Hoppeler, H. (2001) Molecular adaptations in human skeletal muscle to endurance training under simulated hypoxic conditions. Journal of Applied Physiology, 91, 173-182.

Wehrlin, J. P., \& Marti, B. (2006) Live high-train low associated with increased haemoglobin mass as preparation for the 2003 World Championships in two native European world class runners. British Journal of Sports Medicine, 40, e3; discussion e3.

Wilber, R. L. (2001) Current trends in altitude training. Sports Medicine, 31, 249-265. http://dx.doi.org/10.2165/00007256-200131040-00002

Wilber, R. L. (2007) Application of altitude/hypoxic training by elite athletes. Medicine and Science in Sports and Exercise, 39, 1610-1624. http://dx.doi.org/10.1249/mss.0b013e3180de49e6

Wilber, R. L.; Stray-Gundersen, J., \& Levine, B. D. (2007) Effect of hypoxic "dose" on physiological responses and sea-level performance. Medicine and Science in Sports and Exercise, 39, 1590-1599.

http://dx.doi.org/10.1249/mss.0b013e3180de49bd

Zoll, J.; Ponsot, E.; Dufour, S.; Doutreleau, S.; Ventura-Clapier, R.; Vogt, M.; Hoppeler, H.; Richard, R., \& Fluck, M. (2006) Exercise training in normobaric hypoxia in endurance runners. III. Muscular adjustments of selected gene transcripts. Journal of Applied Physiology, 100, 1258-1266.

http://dx.doi.org/10.1152/japplphysiol.00359.2005 